OPEN ACCESS

Edited by: Giovanna Schiavoni, Istituto Superiore di Sanità, Italy

Reviewed by: Alessandra Mancino, San Raffaele Hospital (IRCCS), Italy Maria Cristina Gauzzi, Istituto Superiore di Sanità, Italy

*Correspondence: Zsofia Agod agodzs@gmail.com; Arpad Lanyi alanyi@med.unideb.hu

Specialty section: This article was submitted to Antigen Presenting Cell Biology,

a section of the journal

Frontiers in Immunology

Received: 17 October 2017 Accepted: 10 January 2018

Published: 26 January 2018

Citation:

Agod Z, Pazmandi K, Bencze D,

Vereb G, Biro T, Szabo A, Rajnavolgyi E, Bacsi A, Engel $P$ and Lanyi A (2018) Signaling Lymphocyte Activation Molecule Family 5

Enhances Autophagy and FineTunes Cytokine Response in Monocyte-Derived Dendritic Cells via Stabilization of Interferon Regulatory Factor 8.

Front. Immunol. 9:62. doi: 10.3389/fimmu.2018.00062

\section{Signaling Lymphocyte Activation} Molecule Family 5 Enhances Autophagy and Fine-Tunes Cytokine Response in Monocyte-Derived Dendritic Cells via Stabilization of Interferon Regulatory Factor 8

\author{
Zsofia Agod ${ }^{1 *}$, Kitti Pazmandi', Dora Bencze', Gyorgy Vereb², Tamas Biro', \\ Attila Szabo ${ }^{1,3}$, Eva Rajnavolgyi ${ }^{1,3}$, Attila Bacsi ${ }^{1,3}$, Pablo Engel ${ }^{4}$ and Arpad Lanyi ${ }^{1,3 *}$
}

\begin{abstract}
${ }^{1}$ Department of Immunology, Faculty of Medicine, University of Debrecen, Debrecen, Hungary, ${ }^{2}$ Department of Biophysics and Cell Biology, Faculty of Medicine, University of Debrecen, Debrecen, Hungary, ${ }^{3}$ Department of Bioengineering, Sapientia Hungarian University of Transylvania, Cluj-Napoca, Romania, ${ }^{4}$ Department of Biomedical Sciences, Medical School, University of Barcelona, Barcelona, Spain
\end{abstract}

Signaling lymphocyte activation molecule family (SLAMF) receptors are essential regulators of innate and adaptive immune responses. The function of SLAMF5/CD84, a family member with almost ubiquitous expression within the hematopoietic lineage is poorly defined. In this article, we provide evidence that in human monocyte-derived dendritic cells (moDCs) SLAMF5 increases autophagy, a degradative pathway, which is highly active in dendritic cells (DCs) and plays a critical role in orchestration of the immune response. While investigating the underlying mechanism, we found that SLAMF5 inhibited proteolytic degradation of interferon regulatory factor 8 (IRF8) a master regulator of the autophagy process by a mechanism dependent on the E3-ubiquitin ligase tripartite motif-containing protein 21 (TRIM21). Furthermore, we demonstrate that SLAMF5 influences the ratio of $\mathrm{CD}_{1} \mathrm{a}^{+}$cells in differentiating DCs and partakes in the regulation of IL-1 $\beta, \mathrm{IL}-23$, and IL-12 production in LPS/IFN $\gamma$-activated moDCs in a manner that is consistent with its effect on IRF8 stability. In summary, our experiments identified SLAMF5 as a novel cell surface receptor modulator of autophagy and revealed an unexpected link between the SLAMF and IRF8 signaling pathways, both implicated in multiple human pathologies.

Keywords: SLAMF5, autophagy, dendritic cell, IRF8, TRIM21, IL-12p70, LPS/IFN $\gamma$

\section{INTRODUCTION}

Macroautophagy (here referred to as autophagy) is a conserved catabolic pathway, whereby cytosolic contents are sequestered by de novo formed double-membrane-bound vesicles, called autophagosomes and carried to lysosomes for degradation. It is active at basal levels in most cell types to recycle macromolecules $(1,2)$ and to prevent accumulation of cytotoxic metabolites (3). 
Beyond maintaining cellular homeostasis, autophagy improves cell autonomous and host defense mechanisms against a number of pathogens by regulating intracellular protein trafficking and degradation as well as antigen presentation $(4,5)$. In addition, autophagy guards against both untimely and excessive inflammatory reactions by influencing the activation and duration of inflammation via suppression of ROS accumulation and removal of danger signals as well as regulation of pro-inflammatory cytokine production (6).

Dendritic cells (DCs) continuously migrate from tissues to lymph nodes to present antigens to antigen-specific T cells. The DC pool of non-lymphoid organs is maintained by constant replenishment from circulating monocytes $(7,8)$ whose differentiation into DCs is dependent on the induction of autophagy (9). DCs exploit autophagy to display cytoplasmic self- or foreign antigens on MHC II molecules for CD4 ${ }^{+} \mathrm{T}$ cells (10). This mechanism, depending on the presence or absence of danger signal-induced co-stimulation, contributes to the initiation of a pathogen-specific immune response and to establishment or maintenance of peripheral tolerance, respectively (11). The rate of autophagy therefore must be stringently controlled to adapt to the actual immune context. Stimulation of DCs by LPS has been shown to transiently reduce autophagy and its associated functions (12), presumably to diminish presentation of selfantigens and focus the immune response against an emerging environmental threat. However, as all immune responses, including TLR-mediated functions have the potential to convey damage to host tissues, the recovery of autophagy, reestablishing its anti-inflammatory effects is increasingly recognized as an essential component of the maintenance of host tissue integrity.

Recent work of the Ozato laboratory identified interferon regulatory factor 8 (IRF8) as a positive regulator of autophagy in murine macrophages and DCs exposed to various stress signals, including starvation, exposure to TLR ligands or infection with Listeria monocytogenes (13). Furthermore, their earlier work demonstrated that stimulation of murine macrophages with LPS/FN $\gamma$ induced secretion of IL-12 that was fully dependent on IRF8 (14). The amount and activity of the IRF8 protein were found to be controlled by ubiquitin ligases (TRIM21, c-Cbl), the p62 ubiquitin-binding protein (Sequestosome-1) as well as the deubiquitinase USP4, regulating its proteasomal degradation (15-18). The tasks of IRF8 as a regulator of autophagy or its role in human monocyte-derived dendritic cells (moDCs) functions have not been properly addressed.

Members of the cell surface-expressed signaling lymphocyte activation molecule family (SLAMF) receptors (19-21) have been shown to regulate autophagy. SLAMF1 (CD150) and SLAMF4 (CD244 or 2B4) were reported to bind to the Beclin-1/Vps34 autophagy-associated complex (22-24) responsible for generation of $\mathrm{PI}(3) \mathrm{P}$, a phospholipid involved in autophagic vesicle nucleation. SLAMF1 increased the autophagic flux in human chronic lymphocytic leukemia cells (25) via stabilization of the above autophagic macrocomplex. On the contrary, SLAMF4 was identified as an inhibitor of starvation- and rapamycin-induced autophagy in human lymphoblastoid cell lines and in murine bone marrow-derived macrophages via reducing Vps34 lipid kinase activity (23).
SLAMF5 is a self-ligand receptor broadly expressed on the surface of hematopoietic cells that during cell-cell communication acts both as an adhesion and signaling molecule (26-28). Although its cell surface expression on both the myeloid and plasmacytoid subsets of DCs have been established $(29,30)$, its function in these cells has not been addressed.

Overall, regulatory circuits of autophagy and inflammation are interconnected at multiple levels (4-6), thus molecules involved in the regulation of autophagy have a major impact on the outcome of the immune response. Identification of autophagy regulators, cell surface receptors, readily accessible for antibodies in particular, may provide excellent targets to modulate autophagy in various disease states.

In this report, we examined the effect of SLAMF5 on human moDC responses induced by LPS/IFN $\gamma$, frequently used as a model of infection with Gram-negative bacteria. We identify SLAMF5 as a novel cell surface-expressed regulator of basal autophagy that responding to inflammatory signals fine-tunes cytokine production of human moDCs via affecting a pathway responsible for proteasomal degradation of IRF8.

\section{MATERIALS AND METHODS}

\section{In Vitro Differentiation of moDC and RNA Interference}

Monocytes were isolated from human leukocyte concentrates (buffy coats) obtained from healthy blood donors drawn at the Regional Blood Center of Hungarian National Blood Transfusion Service (Debrecen, Hungary) in accordance with the written approval of the Director of the National Blood Transfusion Service and the Regional and Institutional Ethics Committee of the University of Debrecen, Faculty of Medicine (Debrecen, Hungary). Peripheral blood mononuclear cells (PBMC) were separated by Ficoll Paque (GE Healthcare) gradient centrifugation, and then monocytes were purified by magnetic cell isolation using CD14 antibody-coated magnetic microbeads (Miltenyi Biotech) according to the manufacturer's protocol. The following siRNAs were used for gene silencing by transfection of freshly isolated primary human monocytes:

\section{SLAMF5 sense: 5'-UGGCUAUGUUCUUUCUGCUUGUUC $\mathrm{U}-3^{\prime}$, \\ SLAMF5 antisense: 5'-AGAACAAGCAGAAAGAACAUAGC CA-3', \\ Negative control for SLAMF5 sense: 5'-UGGUAUGCUUUCUG UUCGUUUCUCU-3', \\ Negative control for SLAMF5 antisense: 5'-AGAGAAACGAA CAGAAAGCAUACCA-3'.}

(Stealth ${ }^{\mathrm{TM}}$ RNAi, ThermoFisher Scientific), IRF8 Silencer Select siRNA (Assay ID: s7100), TRIM21 Silencer Select siRNA (Assay ID: s13462), and non-targeted Silencer Select Negative Control No 1. siRNA (ThermoFisher Scientific). The siRNA duplexes were delivered by electroporation using GenePulser Xcell instrument (Bio-Rad Laboratories). Following transfection, monocytes were cultured at a density of $10^{6}$ cells $\mathrm{ml}^{-1}$ on 24 -well plates in complete RPMI-1640 medium containing 10\% fetal 
bovine serum (both from ThermoFisher Scientific), $100 \mathrm{U} \mathrm{ml}^{-1}$ penicillin and $100 \mathrm{ng} \mathrm{ml}^{-1}$ streptomycin (both from SigmaAldrich), supplemented with $80 \mathrm{ng} \mathrm{ml}^{-1}$ GM-CSF (Gentaur Molecular Products), and $100 \mathrm{ng} \mathrm{ml}^{-1}$ IL-4 (PeproTech) for 5 days to generate moDCs. Culture medium was replenished on day 2 by removing three-quarters of the supernatant and replacing it by complete medium containing GM-CSF and IL- 4 .

\section{Receptor Cross-linking}

For SLAMF5 stimulation moDCs adjusted to $10^{7} \mathrm{ml}^{-1}$ were suspended in complete RPMI-1640 medium containing $10 \mu \mathrm{g} \mathrm{ml}^{-1}$ anti-SLAMF5 antibody (clone 152-1D5; LifeSpan BioSciences, Cat. No. LS-C134663) or an IgG isotype control antibody (BioLegend, Cat. No. 400124) at $4^{\circ} \mathrm{C}$ for $45 \mathrm{~min}$. Thereafter, following thorough washing procedures, cells were reseeded into 24-well cell culture plates and incubated in complete medium in the presence of $10 \mu \mathrm{g} \mathrm{ml}^{-1} \mathrm{~F}\left(\mathrm{ab}^{\prime}\right)_{2}$ of goat anti-mouse IgG (Jackson ImmunoResearch Laboratories, Cat. No. 115-006-062) at $37^{\circ} \mathrm{C}$ for $2 \mathrm{~h}$.

\section{Cell Stimulation}

Dendritic cell maturation was induced by simultaneous addition of $100 \mathrm{ng} \mathrm{ml}^{-1}$ LPS (ultrapure lipopolysaccharide from Salmonella minnesota R595, Cat. No. tlrl-smlps) and $10 \mathrm{ng} \mathrm{ml}^{-1}$ recombinant human IFN $\gamma$ (PeproTech, Cat. No. 300-02) for the indicated time periods. For autophagy induction, moDCs were exposed to $50 \mathrm{nM}$ rapamycin (Merck, Cat. No. 553210) for $4 \mathrm{~h}$. The optimal concentration of rapamycin was determined as one that readily increased LC3-II levels without significant toxicity. In some experiments, cells were incubated with $20 \mathrm{nM}$ bafilomycin A1 (BafA1) (InvivoGen, Cat. No. tlrl-baf1) or $1 \mu \mathrm{M}$ MG132 (SelleckChem, Cat. No. S2619) for the last $2 \mathrm{~h}$.

\section{Western Blot Analysis}

For western blot analysis protein extracts were obtained by lysing cells in Laemmli buffer. The samples were resolved by SDS-PAGE and transferred electrophoretically onto nitrocellulose membranes (Bio-Rad Laboratories). Non-specific binding was blocked by TBS-Tween-5\% non-fat dry milk, and then the membrane was probed with anti-SLAMF5 (clone H128), anti- $\beta$-actin (Cat. No. sc-47778), anti-Akt1 (Cat. No. sc-5298), anti-ubiquitin (Cat. No. sc-9133), and anti-TRIM21 (Cat. No. sc-25351) all from Santa Cruz Biotechnology, anti-phosphop70S6K Thr389 (Cat. No. 9206), anti-p70S6K (Cat. No. 9202), and anti-IRF8 (Cat. No. 5628S) all from Cell Signaling, anti-phospho-Akt Ser473 (R\&D Systems, Cat. No. AF887), anti-EAT-2 (LifeSpan BioSciences, Cat. No. LS-C169054) or anti-LC3 (Novus Biologicals, Cat. No. NB100-2220) antibodies followed by washing and incubation with anti-mouse or anti-rabbit horseradish peroxidase-conjugated secondary antibodies (GE Healthcare). Specific signals were detected on X-ray films using the ECL system (SuperSignal West Pico/ Femto chemiluminescent substrate; ThermoFisher Scientific). Protein bands were scanned, and band densities were determined using Kodak 1D Image Analysis software, version 3.6. Relative density was calculated by normalizing to $\beta$-actin band intensities. The level of phosphorylation was normalized to the total amount of the same protein present in the samples.

\section{RNA Extraction, Reverse Transcription and Real-time Quantitative PCR}

Extraction of total RNA was performed using TRI-Reagent (Molecular Research Center) according to the protocol of the manufacturer. Total RNA was treated with DNase I (ThermoFisher Scientific), and then cDNA was synthesized using the High Capacity cDNA Reverse Transcription kit of Applied Biosystems. Quantitative PCR was performed with the ABI StepOne Real-Time PCR System using Dream Taq DNA Polymerase (ThermoFisher Scientific) and gene-specific assay for IRF8 (ThermoFisher Scientific, Assay ID: Hs00175238_m1) according to the manufacturer's instructions. The cycle threshold (Ct) values were determined using the StepOne v2.1 Software (Applied Biosystems). The relative amount of mRNA $\left(2^{-\Delta \mathrm{Ct}}\right)$ was obtained by normalizing to the cyclophilin housekeeping gene in each experiment.

\section{Flow Cytometry}

Viability of the cells was determined on day 5 by 7 -aminoactinomycin-D (7-AAD $10 \mu \mathrm{g} \mathrm{ml}^{-1}$; Sigma-Aldrich) staining for $15 \mathrm{~min}$ immediately before flow cytometry. Cell surface protein expression was detected with FITC-labeled monoclonal antibodies against HLA-A, B, C (Sony Biotechnology), HLA-DQ, HLA-DR, and CD40 (all from BioLegend), PE-labeled monoclonal antibodies against SLAMF5 (BioLegend, clone 1.21), CD14 and CD86 (both from R\&D Systems), DC-SIGN (Sony Biotechnology), and APC-labeled monoclonal antibodies against CD1a (BioLegend). Isotype-matched control antibodies were obtained from BioLegend. Measurement of autophagy was performed using the CYTO-ID Autophagy detection kit (Enzo Life Sciences) according to the manufacturer's instructions. Briefly, $2 \times 10^{5}$ cells were incubated with CYTO-ID Green autophagy detection dye $(1: 2,000)$ for $30 \mathrm{~min}$ at $37^{\circ} \mathrm{C}$, then cells were washed and immediately subjected to flow cytometry. Fluorescence intensities were measured with a FACS Calibur cytometer (BD Biosciences) excluding cell debris by forward/side scatter gating. In case of CD1a analysis, the positive gate was set excluding the area staining with the isotype-matched control $\mathrm{mAb}$. Relative fluorescence and the percentage of positive cells in samples were determined with the FlowJo software.

\section{Confocal Microscopy}

For live cell confocal microscopy, $2 \times 10^{5}$ cells were cultured on 8 -well $\mu$-Slides (chambered coverslip, ibidi GmbH). Where indicated, $50 \mu \mathrm{M}$ Chloroquine (Enzo Life Sciences) was used to inhibit autophagosome turnover. Cells were stained with CYTO-ID Green Detection Reagent $(1: 2,000)$ and Hoechst 33342 Nuclear Stain $(1: 2,000)$ for $30 \mathrm{~min}$ at $37^{\circ} \mathrm{C}$. Next, cells were washed as recommended by the manufacturer and visualized immediately by a Zeiss LSM880 confocal microscope equipped with an $\mathrm{NA}=1.2,40 \times$ water immersion objective. Hoechst was excited at $405 \mathrm{~nm}$ and detected in the $410-485 \mathrm{~nm}$ range, while CYTO-ID was excited at $488 \mathrm{~nm}$ and detected in the $499-562 \mathrm{~nm}$ 
range. Scanning was done at a $0.86 \mu$ s/pixel rate in multitrack/ line switch mode to avoid spectral spillover. Pinhole was set to $32 \mu \mathrm{m}$, equivalent to 1 Airy unit. Lateral $(x-y)$ sampling was $0.87 \mu \mathrm{m} /$ pixel. Images were obtained within $45 \mathrm{~min}$ after staining to avoid loss of fluorescence due to leakage of the dye.

\section{ELISA}

The concentrations of IL- $1 \beta$ and IL-12 in culture supernatants were determined by BD-OptEIA Human ELISA kits (BD Biosciences), and the level of IL-23 was measured with the human IL-23 ELISA Ready-Set Go kit (eBioscience) according to the manufacturer's instructions. Absorbance measurements were carried out by a Synergy HT microplate reader at $450 \mathrm{~nm}$.

\section{Statistical Analysis}

We performed parallel experiments and compared matching observations on control and silenced cells, thus the research design meets the assumptions of the paired $t$-test. However, following monocyte separation, we randomly assigned cells from the same donor to either the knockdown or control groups, cultured the transfected cells separately for 5 days, and then acquired post-intervention data from the two populations. For these reasons, we considered our samples independent and determined the statistical differences between the experimental groups by unpaired two-tailed Student's $t$-test. $p$-values of $<0.05$ were considered to be statistically significant. All data were derived from at least three independent experiments and are expressed as mean \pm SD. Data were analyzed with the Prism software (GraphPad).

\section{RESULTS}

\section{SLAMF5 Is Upregulated during Differentiation and Activation of Human moDCs}

In the process of in vitro differentiation of moDCs, expressions of several receptors with immune modulatory function are upregulated. We found that SLAMF5 is strongly induced when monocytes are differentiated into DCs by GM-CSF and IL-4 suggesting that SLAMF5 is required for the proper functions of immature DCs (Figure 1A). To evaluate the impact of SLAMF5-SLAMF5 interaction on moDC functions, we performed RNA interference targeting SLAMF5 on freshly isolated monocytes. Based on flow cytometry and western blot analyses, SLAMF5 expression was reduced by $80-95 \%$, and silencing was maintained throughout the entire course of differentiation (Figure 1B). Although Binsky et al. have recently published that SLAMF5 acts as a survival receptor in chronic lymphocytic leukemia cells and its downregulation results in receptor induced cell death (31), under our experimental conditions neither the viability of moDCs nor the yield of differentiation was affected by SLAMF5 silencing (Figure 1C). Next, we analyzed whether SLAMF5-deficient cells display any defects in moDC differentiation by measuring CD14, highly expressed on monocytes but downregulated on moDCs, as well as DC-SIGN (CD209), typically expressed by differentiated DCs. As shown in
Figure 1D, cell surface expression of CD14 and DC-SIGN was identical in SLAMF5-silenced and control moDCs indicating that even grossly diminished levels of SLAMF5 did not affect the differentiation of monocytes into moDCs.

As simultaneous LPS and IFN $\gamma$ stimuli further increased its expression level in moDCs (Figure 1A), we analyzed the potential role of SLAMF5 as a modulator of DC activation in response to LPS/IFN $\gamma$. Downregulation of SLAMF5 expression nevertheless was not accompanied by severely impaired LPS/IFN $\gamma$-response as SLAMF5-silenced moDCs retained their capacity to enhance cell surface expression of markers associated with DC maturation in response to LPS/IFN $\gamma$ stimulation. The amounts of HLA-A, B, $\mathrm{C}$ and HLA-DQ increased to the same extent after stimulation of control and SLAMF5-silenced cells. In addition, the expression of the co-stimulatory receptors CD40 and CD86 was identical in control and knockdown cells (Figure S1A in Supplementary Material). Interestingly, silencing of SLAMF5 at an early phase of moDC differentiation led to a moderate, but significant decrease in the subset of CD1a+ DCs (Figure 1E). Further analysis of the above DC-maturation markers in the $\mathrm{CD}^{+} \mathrm{a}^{+}$and $\mathrm{CD} 1 \mathrm{a}^{-}$ DC-subsets, however, revealed no significant differences in response to SLAMF5 silencing (Figures S1B-D in Supplementary Material).

\section{SLAMF5 Enhances Autophagy in moDCs}

In light of the recently established link between autophagy and SLAMF receptors $(23,25)$, we examined whether SLAMF5 partakes in the process of autophagy in resting or activated DCs. Microtubule associated protein 1 light chain 3 beta (LC3 hereafter) is widely used to monitor autophagy. During autophagy induction the processed LC3 is covalently coupled with phosphatidylethanolamine in the newly forming autophagosomal membrane (32). The cleaved cytosolic LC3 (called LC3-I) and the lipid-modified, autophagosome-associated LC3 (LC3-II) can be identified by their different electrophoretic mobility and the level of LC3-II correlates with the amount of autophagosomes (33). As displayed in Figure 2A, we detected lower LC3-II/ $\beta$-actin ratios in SLAMF5-silenced moDCs than in control cells indicating the importance of SLAMF5 in maintenance of basal autophagy. To evaluate kinetic changes in autophagy during DC activation, we treated control and SLAMF5 knockdown moDCs with LPS/ IFN $\gamma$ for 4 and $8 \mathrm{~h}$. The expected transient, activation-dependent decrease in LC3-II levels $(10,12)$ was apparent $4 \mathrm{~h}$ past treatment with LPS/IFN $\gamma$ both in control and SLAMF5-silenced moDCs. At $8 \mathrm{~h}$ past stimulation, however, LC3-II levels recovered in control but not in SLAMF5-silenced cells (Figure 2A; Figure S2A in Supplementary Material). Since LC3-II molecules localized in the inner membrane of autophagosomes are degraded during the autophagic process, lower levels of LC3-II may result from either decreased autophagosome formation or increased autophagic degradation (33). When BafA1, an inhibitor of autophagosome-lysosome fusion and lysosomal acidification (34) was applied LC3-II levels remained lower in the absence of SLAMF5 compared with controls (Figure 2B). As a control for the activity of BafA1, the extent of LC3-II accumulation is shown side by side in BafA1-treated and untreated samples (Figure S2B in Supplementary Material). This observation implies that rather 

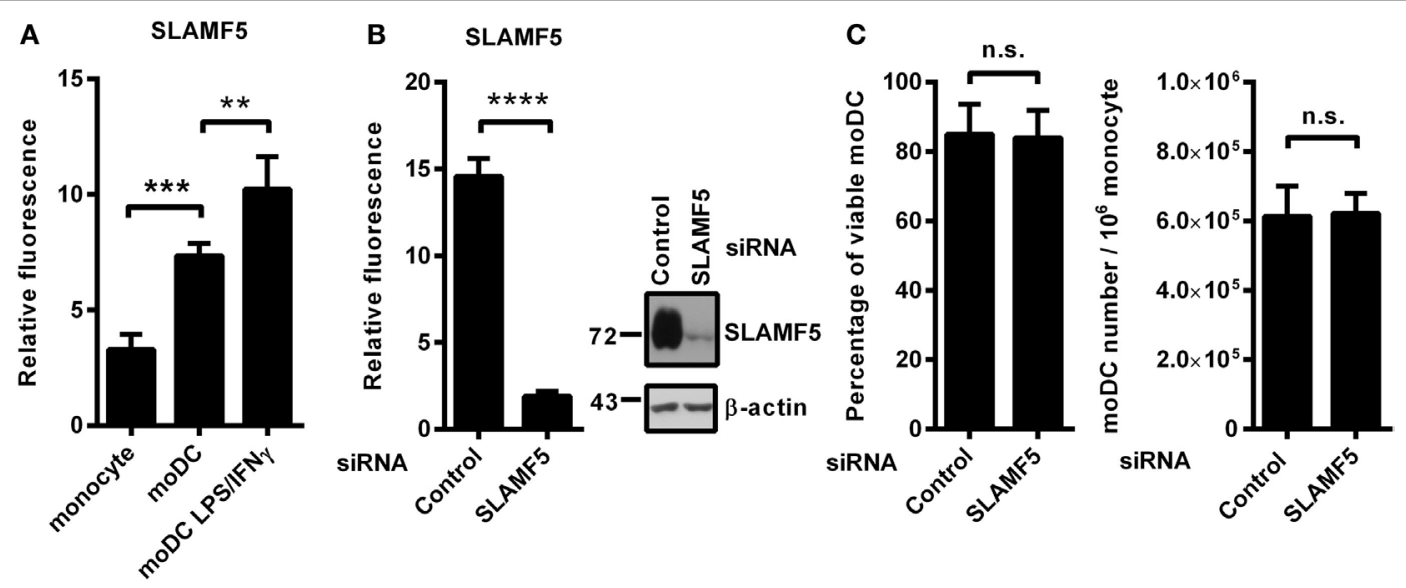

D

Isotype control

$\square$ Control siRNA

口 SLAMF5 siRNA
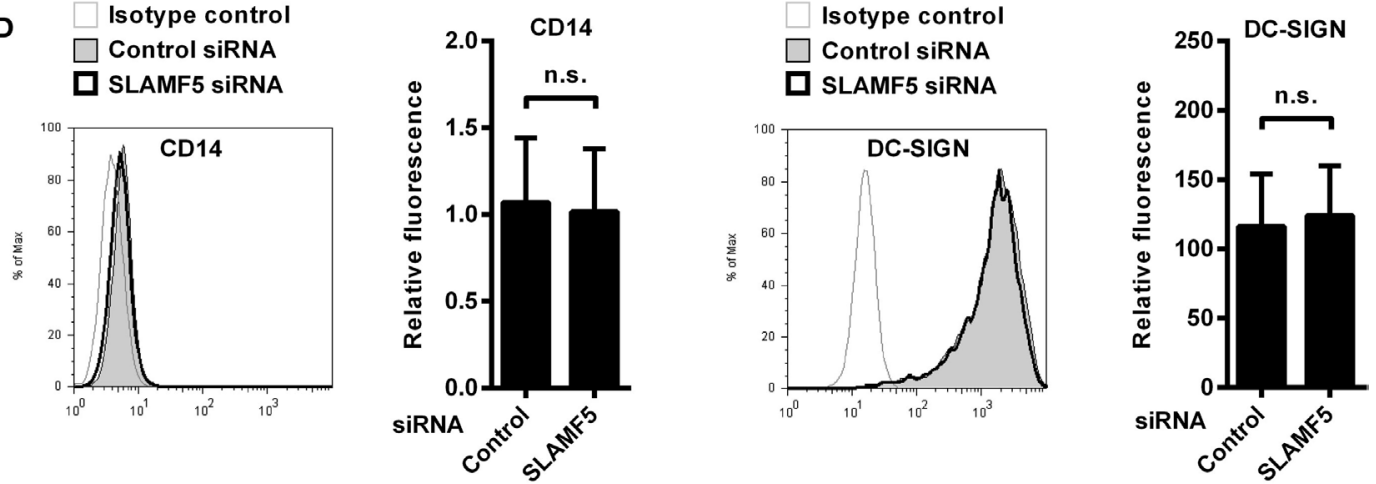

E

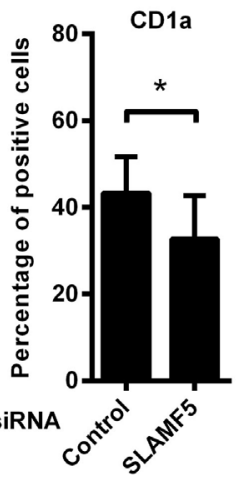

FIGURE 1 | The effect of SLAMF5 silencing on the phenotype of moDCs. (A) Cell surface expression of SLAMF5 on monocytes and on GM-CSF/IL-4-differentiated moDCs treated or not with LPS/IFN $\gamma$ was assessed by flow cytometry. (B) Monocytes were transfected with the indicated siRNAs and differentiated into moDCs. On day 5, SLAMF5 expression was measured by flow cytometry and western blot analysis. $\beta$-actin was used as protein loading control. (C) Viability of control and SLAMF5 knockdown moDCs was determined by 7-aminoactinomycin-D (7-AAD) dye exclusion. Bar graphs indicate percentage of cells negative for 7-AAD (left panel) and the total number of moDCs differentiated from $10^{6}$ monocytes (right panel). (D) Expression levels of CD14 and DC-SIGN in control and SLAMF5 knockdown moDCs were measured by flow cytometry. Representative histograms show protein expression in control (thin line with gray shading) and knockdown cells (bolded black line) or staining with isotype control antibody (thin gray line). Bars show the relative fluorescence intensity values of CD14 and DC-SIGN, calculated using the respective isotype-matched control antibodies. The results shown are taken from four independent donors. (E) Representative histograms show CD1a expression in control (thin line with gray shading) and SLAMF5-silenced (bolded black line) moDCs. Bars show the percentage of CD1a cells. Data are presented as means $\pm \mathrm{SD}$ of six independent experiments. Error bars indicate $\mathrm{SD}\left({ }^{\star} p<0.05,{ }^{* \star} p<0.01,{ }^{* \star *} p<0.001\right.$, and ${ }^{\star \star * *} p<0.0001$; ns, not significant).

than being involved in the maturation process of autophagosomes, SLAMF5 appears to be a positive regulator of autophagosome biogenesis. To further support SLAMF5-mediated regulation of autophagy we used CYTO-ID, a fluorescent dye that selectively labels autophagic vacuoles in live cells (35) and thus can be readily quantified by flow cytometry (36). In agreement with the results obtained by LC3 blotting, fluorescence intensity of CYTO-ID was significantly lower in SLAMF5 knockdown cells 

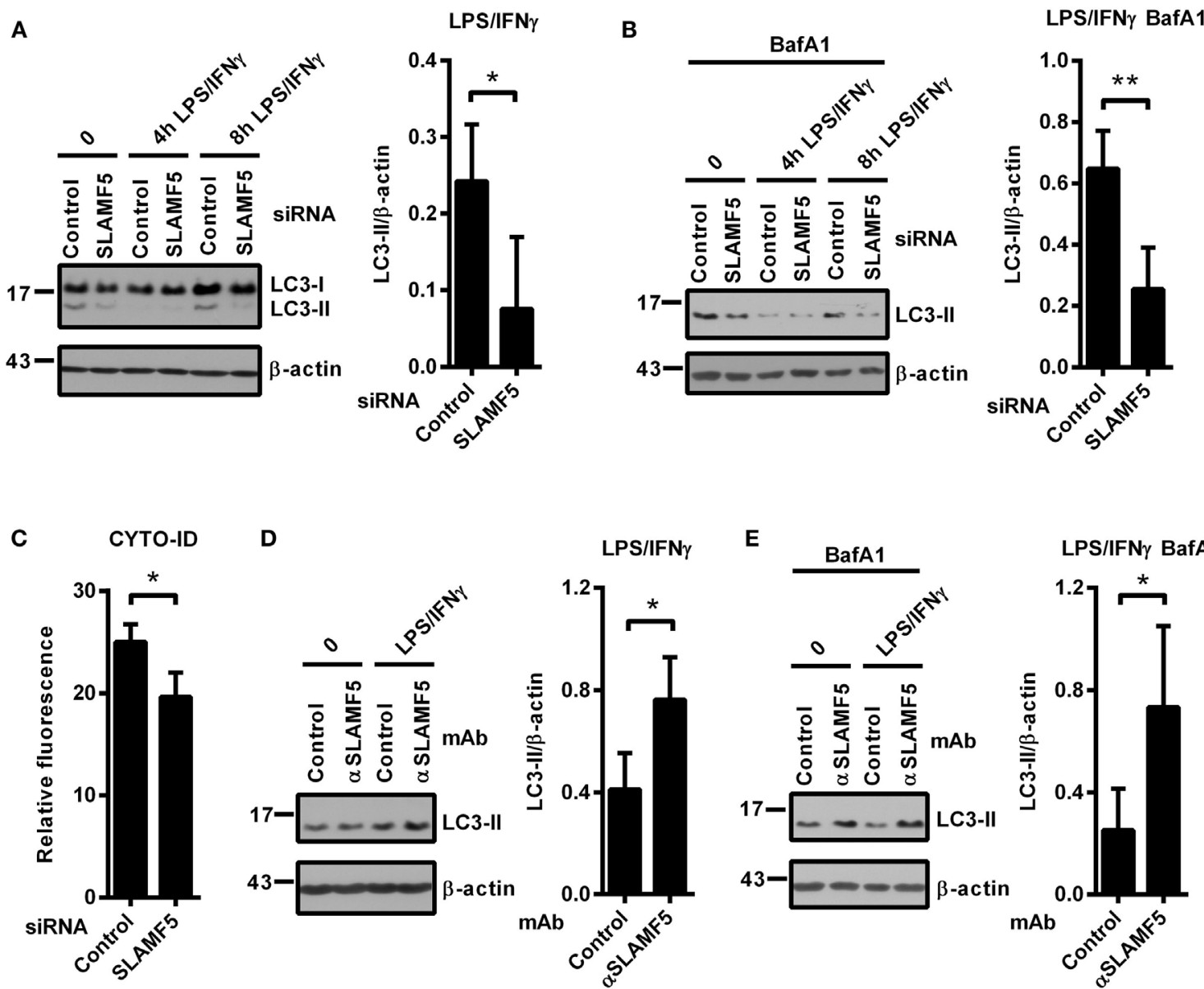

FIGURE 2 | SLAMF5-mediated enhancement of dendritic cell autophagy under steady-state conditions and in response to LPS/IFN $\gamma$ activation. Control and SLAMF5-silenced moDCs were stimulated or not with LPS/IFN $\gamma$ for the indicated time periods in the absence [(A) cropped] or in the presence of 20 nM bafilomycin A1 (BafA1) applied for the last $2 \mathrm{~h}$ (B). Conversion of LC3 was determined by western blotting. A representative immunoblot of four independent experiments is shown on the left; ratios of LC3-II and $\beta$-actin determined by densitometry are shown in the right panels. (C) Control and SLAMF5-depleted moDCs were stained with CYTO-ID. Fluorescence intensity was determined by flow cytometry. Graph depicts the relative fluorescence intensity of CYTO-ID obtained in four independent experiments (D,E). moDCs were conditioned with $10 \mu \mathrm{g} \mathrm{ml}^{-1}$ SLAMF5-specific or control antibodies followed by cross-linking with $10 \mu \mathrm{g} \mathrm{ml}^{-1} \mathrm{~F}(\mathrm{ab})_{2}$ fragment of goat anti-mouse lgG, then stimulated with LPS/IFN $\gamma$ for $8 \mathrm{~h}$ in the absence (D) or in the presence (E) of $20 \mathrm{nM}$ BafA1 applied for the last $2 \mathrm{~h}$ of the experiment. LC3 and $\beta$-actin levels were analyzed by western blotting; a representative blot and the mean ratios of LC3-II to $\beta$-actin from three independent experiments are shown. Data are expressed as the mean $\pm \mathrm{SD}\left({ }^{*} p<0.05\right.$ and $\left.{ }^{* *} p<0.01\right)$.

than in controls (Figure 2C). Confocal microscopy analysis of CYTO-ID staining revealed punctate structures, the number of which increased in the presence of chloroquine (Figure S2C in Supplementary Material). Chloroquine inhibits maturation of autophagosomes by affecting lysosomal acidification, thereby resulting in accumulation of large CYTO-ID positive compartments (37). Therefore, the fluorescence signal detected by flow cytometry indeed resulted from accumulation of the dye in autophagic vacuoles.

If SLAMF5 is an important regulatory module of the autophagy pathway, increased signaling via SLAMF5 is expected to enhance autophagy. To test this, we used the SLAMF5-specific agonistic antibody 152.1D5 reported to induce SLAMF5 signaling both in T cells (38) and in chronic lymphocytic leukemia cells (31). Cross-linking of cell surface SLAMF5 with the 152.1D5 monoclonal antibody greatly increased LC3-II levels both in the presence and absence of BafA1 (Figures 2D,E). To exclude Fc receptor-mediated effects, an isotype-matched $\mathrm{mAb}$ was used as control in each experiment.

Collectively, these data demonstrate that SLAMF5 in moDCs potentiates basal autophagy and the recovery of autophagy after LPS/IFN $\gamma$ activation.

\section{Autophagy Defect Observed in the Absence of SLAMF5 Is Not Mended by Rapamycin}

mTOR is one of the key negative regulators of autophagy (39). One potential mechanism by which SLAMF5 could enhance the level of autophagy was inhibition of the mTORC1 complex. To examine this scenario we used rapamycin (RAPA), a specific inhibitor of the mTORC1 complex and a well-known inducer 
of autophagy (39). Control and SLAMF5-silenced moDCs were treated with rapamycin for $4 \mathrm{~h}$ either in the presence or absence of BafA1. As shown in Figures 3A,B, the autophagy defect remained apparent in SLAMF5-silenced cells even when mTORC1 was suppressed, suggesting that SLAMF5-mediated regulation of autophagy is independent of mTOR activity. To further test this scenario, SLAMF5-silenced and control moDCs were activated with LPS/IFN $\gamma$, and phosphorylation of Akt, a kinase upstream of mTOR, and of the mTOR target protein p70S6K was monitored by western blot analysis. If SLAMF5 increases autophagy by inhibition of mTOR activity, silencing of SLAMF5 should potentiate the Akt/mTOR/p70S6K pathway resulting in increased phosphorylation of these proteins. However, as shown in Figure 3C, the amounts of phospho-Akt and phospho-p70S6K were comparable in SLAMF5-silenced and control cells. The fact that rapamycin failed to restore the autophagy defect present in SLAMF5-silenced cells together with our findings that phosphorylation of Akt, and p70S6K was unaffected at diminished SLAMF5 protein levels strongly suggest that mTOR is not the main mediator of the autophagy pathway modulated by SLAMF5.

\section{SLAMF5 Regulates Autophagy via Preventing Proteasomal Degradation of IRF8}

Recently, Gupta and colleagues reported that IRF8 is expressed under steady-state conditions in murine bone marrow-derived DCs and its expression level is significantly increased in response to LPS/IFN $\gamma$ treatment. Moreover, IRF8 was shown to
A
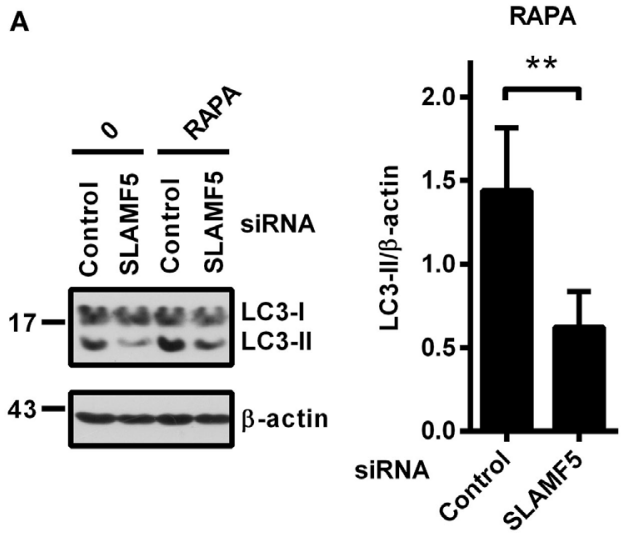

C
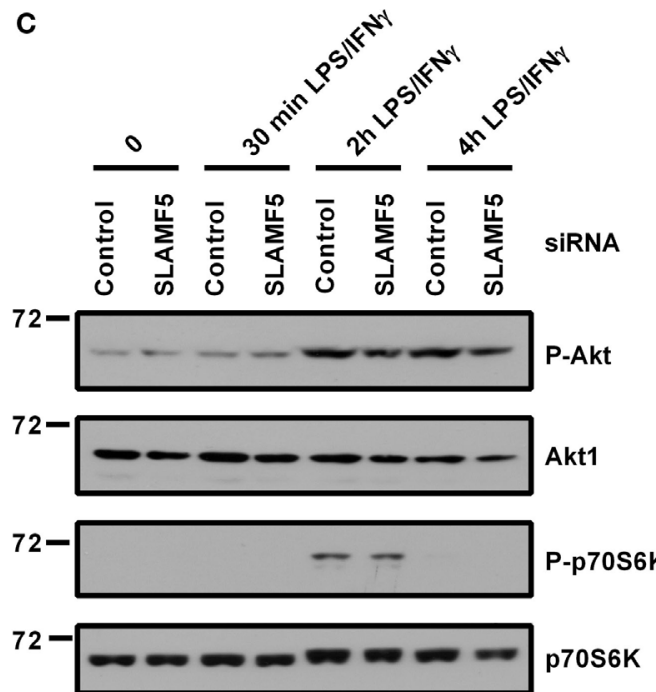

B

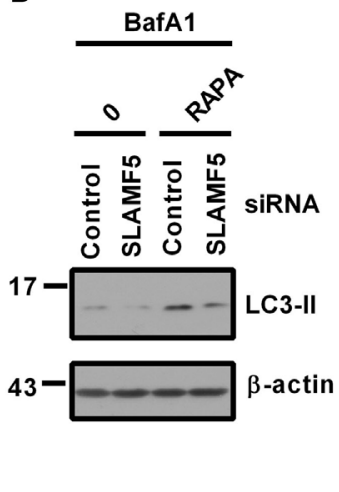

RAPA BafA1

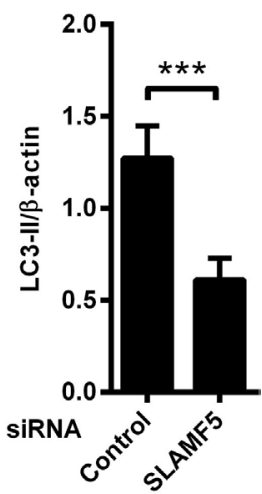

LPS/IFN $\gamma$

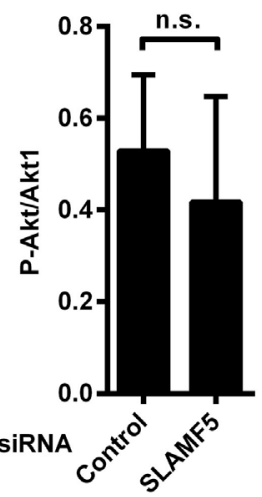

LPS/IFN $\gamma$

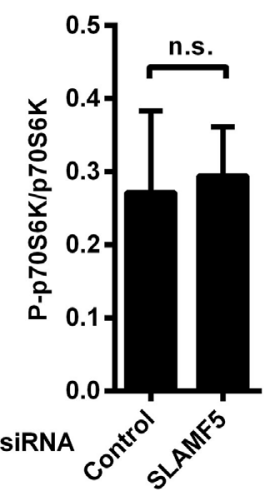

FIGURE 3 | Enhancement of autophagy by SLAMF5 is not mediated by inhibition of the mTORC1 complex. (A) The autophagy-inducer rapamycin was applied to control or SLAMF5-silenced cells for $4 \mathrm{~h}$. Autophagy was monitored by western blot analysis using an LC3-specific antibody. A representative blot of four independent experiments is shown on the left, and densitometry of LC3-II to $\beta$-actin ratios is depicted on the right panel. (B) Cells were treated as described in panel (A), except that they were cultured in the presence of $20 \mathrm{nM}$ bafilomycin A1 (BafA1) for the last $2 \mathrm{~h}$. Data were analyzed as described in panel (A). (C) SLAMF5-silenced or control monocyte-derived dendritic cells were activated with LPS/IFN $\gamma$, and phosphorylation of Akt and p70S6K was analyzed with immunoblotting. Representative blots of four independent experiments are shown. The phospho-proteins were normalized to the total levels of Akt1 and p70S6K. Data are expressed as mean \pm SD $\left({ }^{* *} p<0.01\right.$ and ${ }^{* \star *} p<0.001$; ns, not significant). 
switch on the expression of many genes involved in all phases of autophagy (13). Based on the central role of IRF8 in the process of autophagy, we hypothesized that the molecular mechanism by which SLAMF5 promotes autophagy may involve the regulation of IRF8 activity. To test this, first, we silenced SLAMF5 and analyzed induction of IRF8 expression in response to treatment with LPS/IFN $\gamma$. As shown in Figure 4A, the amount of IRF8 protein in SLAMF5-silenced moDCs was significantly decreased. To gain more insight into the effects of decreased IRF8 levels on moDC differentiation and functions, IRF8 expression was silenced using an IRF8-specific siRNA (Figure 4B). Interestingly, we found that silencing of IRF8, a lineage determining factor for myeloid cells, had no significant effect on the downregulation of CD14 expression or the induction of DC-SIGN expression associated with the differentiation process in the presence of IL-4 and GM-CSF (Figure 4C). Moreover, IRF8 expression was also not requisite for the expression of SLAMF5 that was significantly increased in IRF8-silenced cells (Figure S3 in Supplementary Material).

Importantly, the shift toward $\mathrm{CD}^{-} \mathrm{a}^{-}$moDCs observed in SLAMF5-silenced cells was evident in IRF8 knockdown cells (Figure 4D), consistent with a previous study by Granato et al., reporting that interference with the autophagic process in monocytes resulted in reduced expression of CD1a on moDCs (40). To test whether IRF8 acts as a regulator of autophagy in human moDCs, the intensity of autophagy was compared in IRF8-silenced and control moDCs. In line with its function in murine macrophages and DCs, and its suspected role as a mediator of SLAMF5's effects, IRF8-silenced moDCs showed significantly decreased autophagy compared with controls. As shown in Figures 4E,F, the amount of LC3-II as well as the fluorescence intensity of CYTO-ID were significantly decreased (Figure 4G). These findings demonstrate that similar to murine bone marrow-derived DCs, IRF8 is used for the regulation of autophagy in human moDCs. To determine whether SLAMF5 and IRF8 are part of the same or different autophagy regulatory pathways, SLAMF5 was cross-linked with the above described 152.1D5 mAb antibody on moDCs transfected with control or IRF8-specific siRNA. As shown in Figure $4 \mathbf{H}$, cross-linking of SLAMF5 significantly increased autophagy in moDCs transfected with the control oligonucleotides; however, this induction was dependent on the presence of IRF8. This observation established IRF8 as part, and a downstream element of the SLAMF5 autophagy regulatory pathway.

Protein levels are determined by the rate of transcription followed by translation as well as the rate of protein degradation. When SLAMF5-silenced and control moDCs were activated by LPS/IFN $\gamma$, both the kinetics and the intensity of IRF8-transcription were found to be identical between silenced cells and controls (Figure 5A) indicating the lack of SLAMF5mediated regulation at the level of IRF8-transcription. Given that IRF8 activity and its stability are regulated by polyubiquitination and subsequent proteasomal degradation $(15,17)$, we examined whether degradation of IRF8 by the proteasome was affected by SLAMF5. When moDCs were activated by LPS/IFN $\gamma$ in the presence of the proteasome inhibitor MG132, IRF8 levels in SLAMF5 knockdown moDCs were completely restored (Figure 5B).
As a control, enrichment of ubiquitin-linked proteins in the presence of MG132 was confirmed by probing identical blots with a ubiquitin-specific antibody (Figure S4 in Supplementary Material). Since ubiquitination of IRF8 by the E3-ubiquitin ligases TRIM21 (Ro52) (15) and casitas B-lineage lymphoma (c-Cbl) (17) has been described in murine macrophages, we tested whether IRF8 degradation in SLAMF5-silenced cells required the participation of these ligases. To this end, first, we confirmed induction of TRIM21 in response to LPS/IFN $\gamma$ (Figure S5 in Supplementary Material) and its silencing in the presence of TRIM21 gene-specific siRNA (Figure 5C). Next, SLAMF5 alone, or in combination with TRIM21, was silenced, and the level of IRF8 protein was measured in moDCs treated with LPS/IFN $\gamma$. As shown in Figure 5D, IRF8 degradation in SLAMF5-silenced moDCs was blocked when cells were cotransfected with the TRIM21-specific, but not with the control oligo. This finding clearly showed that increased degradation of IRF8 in the absence of SLAMF5 was dependent on the presence of TRIM21. In similar experiments, IRF8 was not rescued from degradation by silencing the E3-ubiquitin ligase $c$ - $C b l$ (data not shown).

Two single SH2 domain-containing proteins, the SLAMassociated protein and the Ewing sarcoma activated transcript-2 (EAT-2) are major transducers of SLAMF-receptor signaling $(19,41,42)$. Based on its reported expression in antigen-presenting cells (43) and a recent report describing it as an enhancer of the autophagy process (23), EAT-2 emerges as a potential adapter transmitting SLAMF5 signals. However, when EAT-2 expression was thoroughly examined in our model, we found EAT-2 expressed in human monocytes, but abruptly downregulated in response to the used DC differentiation signals. The EAT-2 protein was absent both in resting and LPS/IFN $\gamma$-activated moDCs (Figure S6 in Supplementary Material), indicating that SLAMF5 drives autophagy in moDCs in an EAT-2-independent manner.

Taken together, unlike its induction of gene expression and translation, the stability of the IRF8 protein is strongly affected by the presence of SLAMF5 that appears to control the activity of TRIM21. These data also support a mechanism connecting SLAMF5 to autophagy as part of the regulatory cascade sustaining activity of IRF8, a major transcription factor of autophagyrelated genes.

\section{SLAMF5 and IRF8 Silencing in Monocytes Results in Development of moDCs with Overlapping Changes in Cytokine Secretion}

To further support our hypothesis that the impact of SLAMF5 silencing on DC functions is the consequence of IRF8 degradation, hereinafter, we examined whether depletion of IRF8 could induce similar functional changes to those seen in SLAMF5silenced moDCs. Given the well-characterized role of autophagy in the inflammatory response, the contribution of SLAMF5 and IRF8 to cytokine production of moDCs was examined. To this end, we measured the amount of pro-inflammatory cytokines in the supernatant of control and knockdown moDCs exposed 

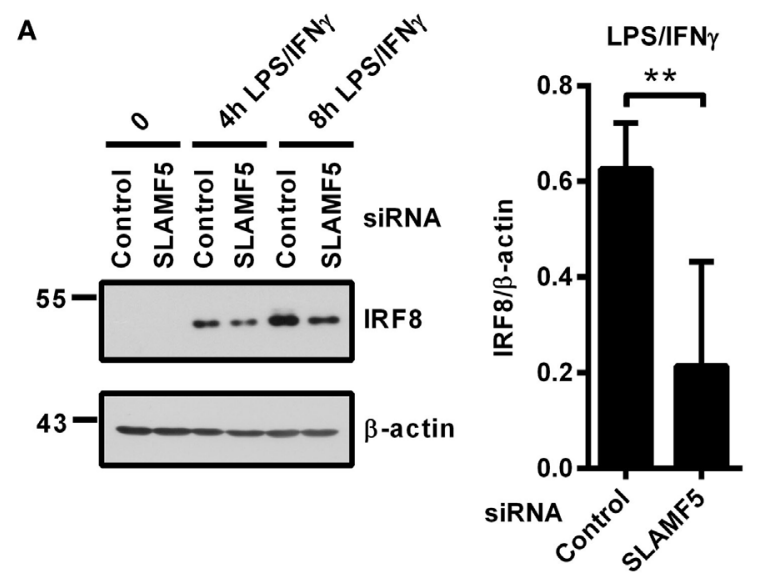

B
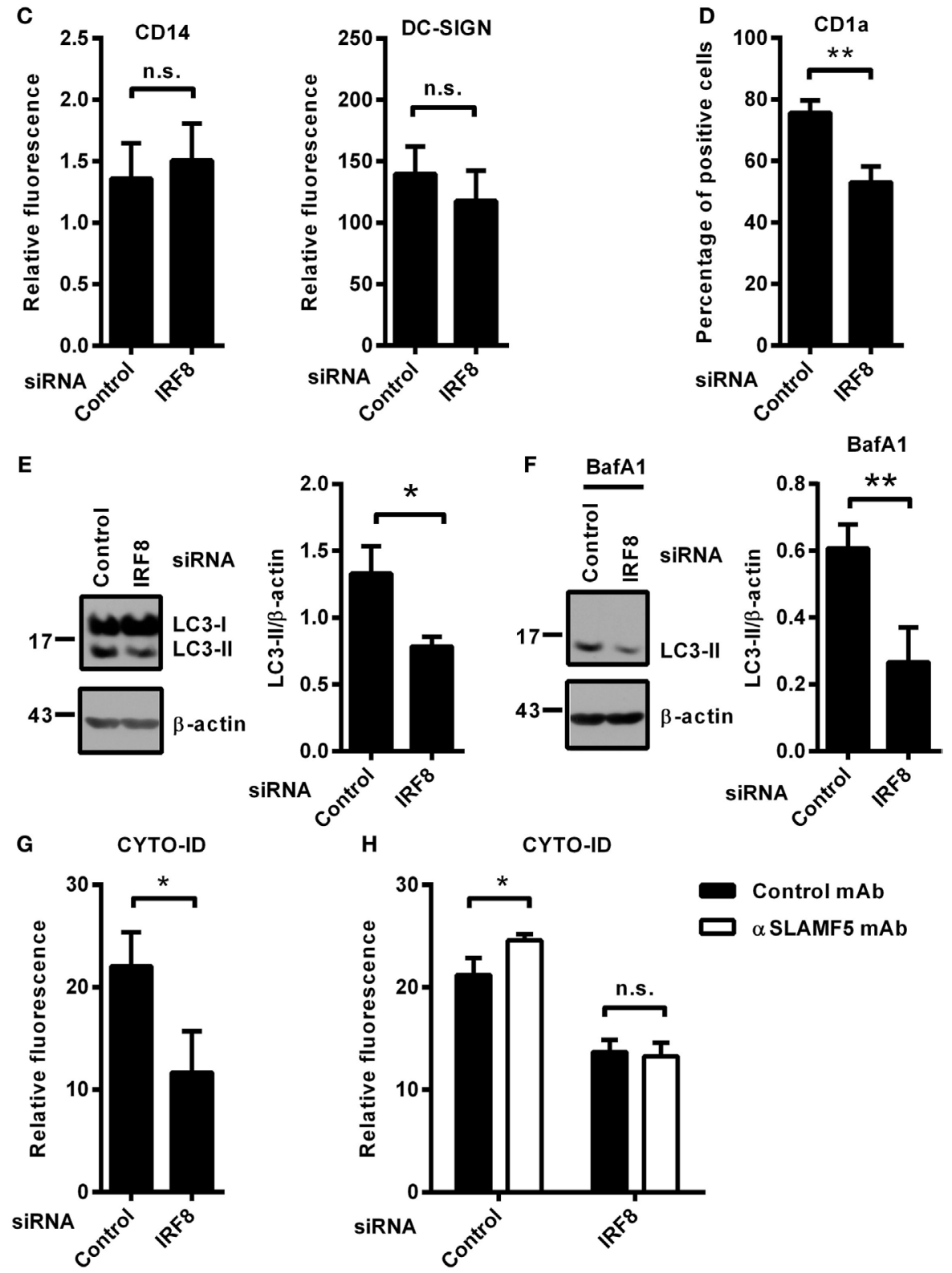
FIGURE 4 | SLAMF5 controls the level of IRF8 protein, a positive regulator of autophagy in moDCs. (A) moDCs transfected with either control or an SLAMF5specific siRNA were treated with LPS $\left(100 \mathrm{ng} \mathrm{ml}^{-1}\right)$ and IFN $\gamma\left(10 \mathrm{ng} \mathrm{ml}^{-1}\right)$ for the indicated time periods. IRF8 protein level was measured by immunoblotting, and the ratio of IRF8/ $\beta$-actin was quantified from five independent experiments. (B) Efficiency of IRF8 knockdown was tested on day 5 by western blot analysis; a representative blot is shown with $\beta$-actin as loading control. (C) Expression levels of CD14 and DC-SIGN as well as CD1a (D) were measured in control and IRF8 knockdown moDCs by flow cytometry. Bars show the relative fluorescence intensity values or the percentage of positive cells. The results shown are taken from three independent experiments. LC3-II expression was measured in control or IRF8-silenced moDCs by immunoblotting in the absence (E) or in the presence of bafilomycin A1 (BafA1) (F). One representative of three experiments is shown. Bar charts display the ratio of LC3-II/ $\beta$-actin. (G) CYTO-ID staining of control and IRF8-silenced moDCs from three donors was analyzed by flow cytometry. (H) CYTO-ID staining of control and IRF8-silenced moDCs in which SLAMF5 was cross-linked with the SLAMF5-specific agonistic antibody 152.1D5 as described in Section "Materials and Methods." Data are expressed as the mean \pm SD $\left({ }^{*} p<0.05\right.$ and ${ }^{* *} p<0.01$, ns, not significant).
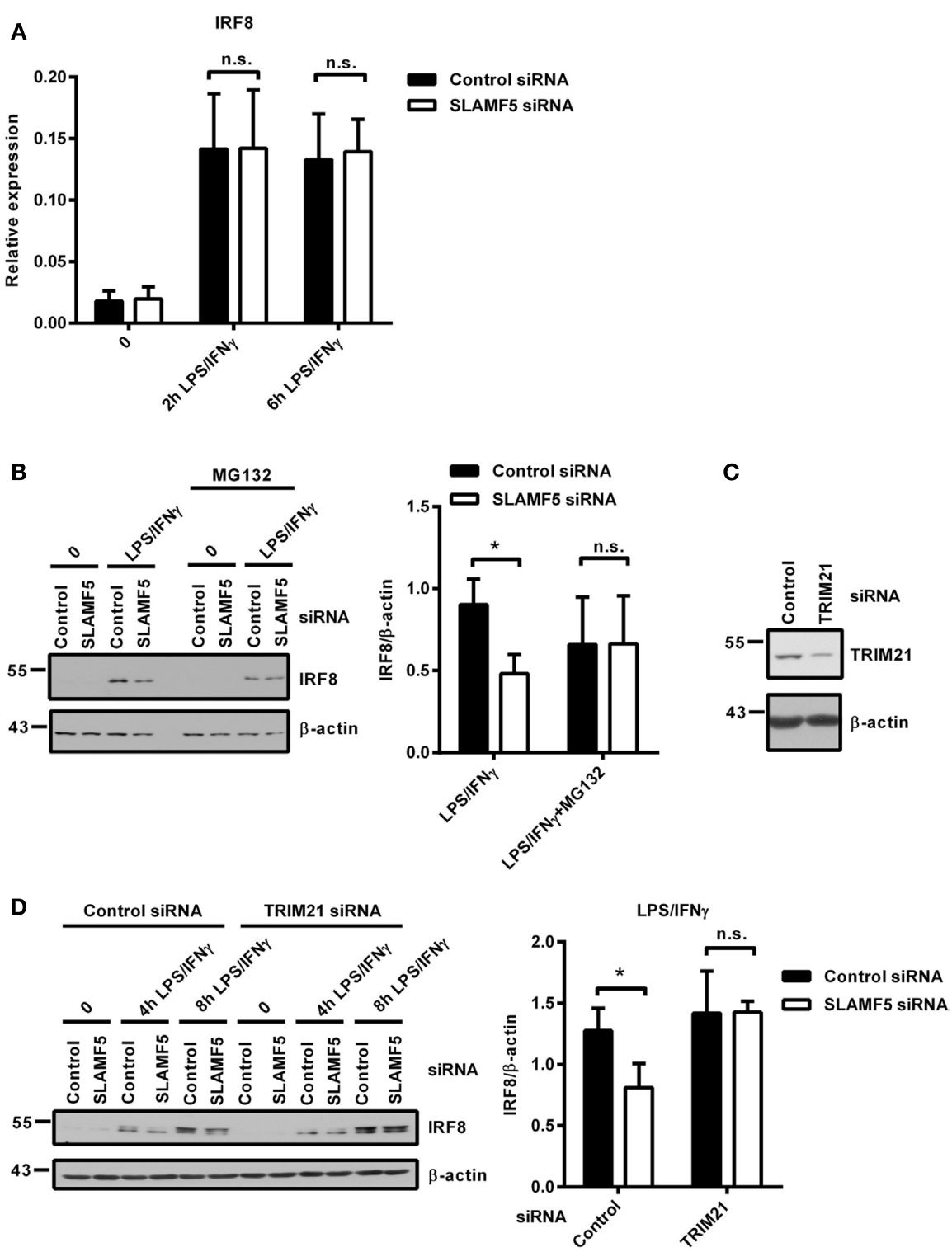

FIGURE 5 | Proteasomal degradation of IRF8 is dependent on the E3-ubiquitin ligase TRIM21. (A) Kinetics of IRF8 mRNA expression were analyzed by quantitative PCR in five donors after treatment of control and SLAMF5 knockdown moDCs with LPS/IFN $\gamma$ for the indicated time periods. (B) Control and SLAMF5 knockdown moDCs were stimulated with LPS/IFNy for $8 \mathrm{~h}$ and, where indicated, $1 \mu \mathrm{M} \mathrm{MG132}$ was added $2 \mathrm{~h}$ before harvesting. A representative immunoblot (left) and densitometric quantification (right) of three immunoblots from independent experiments are shown. (C) Efficiency of TRIM21 knockdown was tested on day 5 by western blot analysis; a representative blot is shown with $\beta$-actin as loading control. (D) SLAMF5 expression alone or together with TRIM21 expression was silenced by transfection of moDCs with the indicated set of gene-specific or control siRNAs. Transfected cells were activated with LPS/IFNy for the indicated time periods. IRF8 protein levels were determined by western blotting. One representative blot (left) and densitometry of three independent experiments (right) are shown. Data are expressed as the mean $\pm \mathrm{SD}\left({ }^{*} p<0.05 ;\right.$ ns, not significant). 
to LPS/IFN $\gamma$. SLAMF5- (Figure 6A) or IRF8-silenced cells (Figure 6B) released significantly higher amounts of IL-1 $\beta$ and IL-23 than did control cells, implying that both SLAMF5 and IRF8 may function to restrain excessive inflammatory reactions. On the contrary, SLAMF5-silenced or IRF8 knockdown cells produced less IL-12 than control cells. Remarkably, these experiments also showed that IL-12 production in response to LPS/IFN $\gamma$ stimulation was fully IRF8 dependent (Figure 6B). The finding that loss of IRF8 replicated several changes in DC phenotype (Figures 1D,E and 4C,D) and cytokine production observed in SLAMF5-silenced cells, lends further support to a model in which SLAMF5 and IRF8 are part of the same regulatory pathway.

Taken together, data presented above identifies SLAMF5 as a novel cell surface-expressed regulator of DC autophagy operating via stabilization of one of the autophagy master-regulator transcription factors, IRF8 (Figure 7).

\section{DISCUSSION}

SLAMF receptors regulate various stages of the immune response including the differentiation of innate and classical $\mathrm{T}$ cell subsets as well as the $\mathrm{T}$ - and $\mathrm{B}$ cell memory response $(19,21,41)$. Consequently, in addition to antimicrobial responses, SLAMF receptors have been shown to contribute to pathological autoimmune responses, i.e., colitis and systemic lupus erythematosus (44-46), as well as to neuropsychiatric diseases with inflammatory and neurodegenerative etiologies (47, 48). Despite its ubiquitous expression in practically all hematopoietic lineages, mice carrying defective SLAMF5 alleles at both chromosomes have a relatively mild phenotype. The main defect appears to be restricted to germinal center formation, specifically due to lack of proper adhesion and functional interaction between $\mathrm{T}$ follicular helper (Tfh) and $\mathrm{B}$ cells, but not DCs (28). However, normal DC functions in the absence
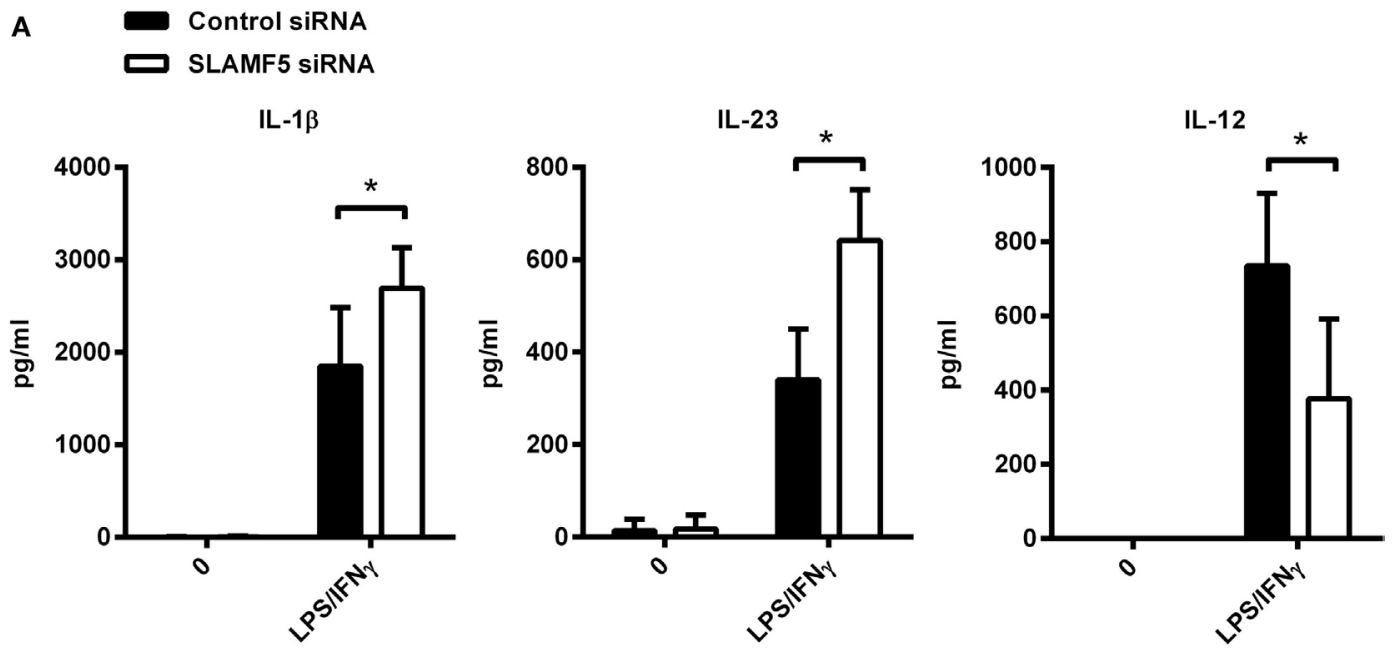

B

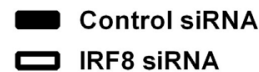

IL-1 $\beta$

IL-23
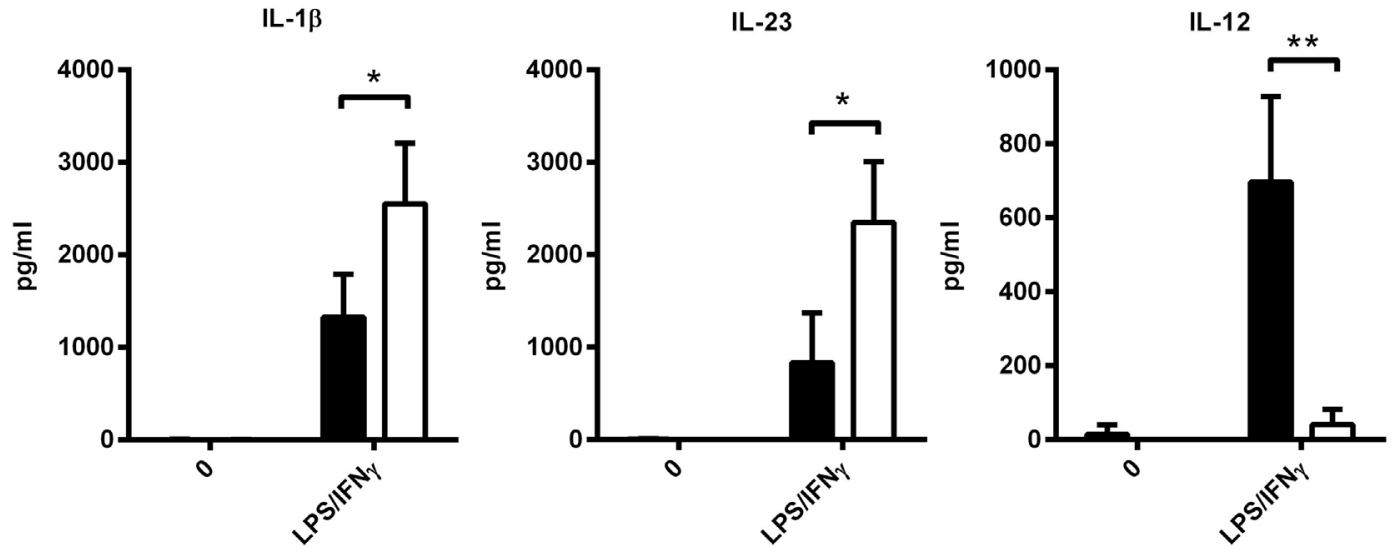

FIGURE 6 | Cytokine production of SLAMF5- and IRF8-silenced moDCs. SLAMF5- (A) or IRF8-silenced (B) moDCs were left untreated or stimulated with LPS/IFN and their cytokine productions were compared with cells transfected with control oligonucleotides. Cytokine concentrations in supernatants were determined at $8 \mathrm{~h}$ (IL-1 $\beta$ and IL-23) or $12 \mathrm{~h}$ (IL-12) by ELISA. Lack of bars indicates cytokine secretion below the limit of sensitivity for the ELISA. Data are presented as means \pm SD of four independent experiments $\left({ }^{*} p<0.05\right.$ and $\left.{ }^{* *} p<0.01\right)$. 


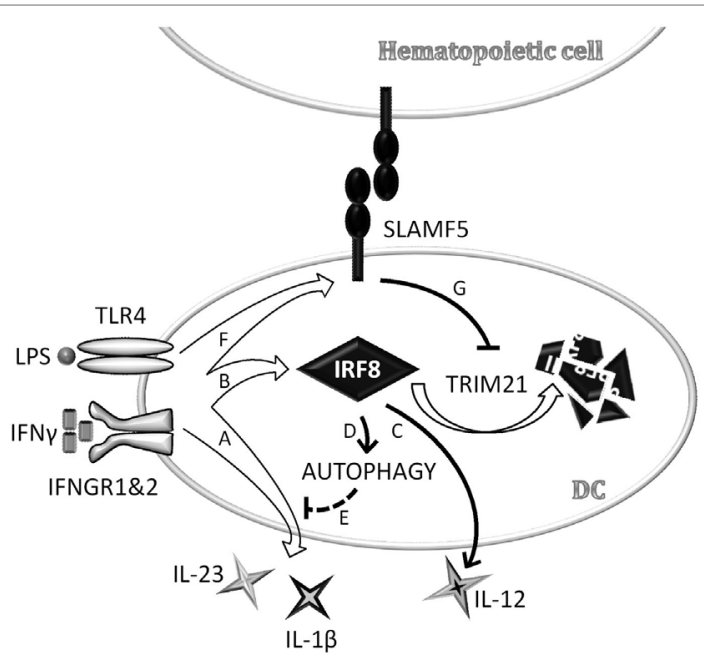

FIGURE 7 | Schematic model depicting signaling lymphocyte activation molecule family (SLAMF) 5-mediated modulation of autophagy and cytokine production in monocyte-derived dendritic cells (moDCs) via stabilization of interferon regulatory factor 8 (IRF8). In an ongoing infection with Gramnegative bacteria, dendritic cells (DCs) are exposed to LPS and IFN $\gamma$ that are known to reduce autophagy, presumably to prevent exposure of autoreactive T cells to self-antigens in the presence of strong co-stimulatory signals. In the early phase of DC activation, concomitant with the secretion of proinflammatory cytokines (A), IRF8 expression is induced (B). IRF8 in turn promotes the production of IL-12 (C) and restores autophagy (D) to control overproduction of potentially self-destructive cytokines, such as IL-1 $\beta$ and IL-23 (E). We found that SLAMF5 is upregulated in response to LPS/IFN $\gamma$ (F) and inhibits TRIM21-mediated proteasomal degradation of IRF8 (G). By this mechanism, SLAMF5 acts as a positive regulator of autophagy in moDC, likely impacting on the outcome of antimicrobial responses as well as on autophagy-driven pathologies.

of SLAMF5 may be explained by compensatory mechanisms implemented by other SLAMF members. Alternatively, appearance of the unique functions of SLAMF5 may require specific environmental challenges, yet to be identified. In support of this latter scenario, SLAMF5 has been shown to regulate TCR-, BCR-, and FCeRI-mediated signal-transduction (26, 28, 49). The function of SLAMF5 in myeloid cells, however, remains poorly understood. In a single report, SLAMF5 knockdown was found to decrease LPS-induced TNF-alpha and IL-6 production of murine bone marrow-derived macrophages (50). In our work, we set up experiments to analyze the function of SLAMF5 in human moDCs. When SLAMF5 expression was decreased by 80-95\% using transfection of specific siRNA, human peripheral blood monocytes readily differentiated into moDCs, suggesting that SLAMF5 may not be required for the main steps of moDC differentiation. In addition, although SLAMF5 was shown to act as a survival factor in certain tumor cell lines (31), viability of SLAMF5-deficient moDCs appeared to be identical to that of control cells, suggesting that the survival-promoting effect of SLAMF5 may be context dependent, lineage-specific, or even restricted to transformed cells.

Nevertheless, production of IL- $1 \beta$ and IL-23 was significantly enhanced by cells with reduced SLAMF5 levels while IL-12 secretion was diminished compared with controls. These functional changes occurred in the absence of altered expression of receptors associated with DC activation including HLA proteins or the co-stimulatory molecules CD40 and CD86. Thus, SLAMF5mediated modulation of cytokine production without affecting co-stimulatory receptor expression may allow DCs to alter polarization of naïve T cells while supporting their proliferation. In this regard, independent regulation of DC maturation and inflammatory cytokine production has been described by the D'Oro group in TLR ligand-activated DCs treated with the panSrc-kinase inhibitor PP1 or PP2 $(51,52)$.

More rigorous analysis of the SLAMF5-deficient moDCs identified a consistent, albeit moderate decrease of CD1a expression that together with an increase in IL-1 $\beta$ and IL-23 production was reminiscent of the phenotype of DCs treated with autophagy inhibitors (40). Inhibition of autophagy, either with the PI3K inhibitor 3-methyladenine or with siRNA against the autophagy protein Atg7 significantly increased IL-1 $\beta$ production of human PBMC stimulated with either LPS (53) or Mycobacterium tuberculosis (Mtb) (54). Likewise, LPS-activated human moDCs produced more IL-23 under such circumstances (55). It has also been reported that IL-1 $\beta$, produced following autophagy inhibition in DCs, acted in an autocrine manner to drive IL-23 secretion and this appears to be the mechanism through which autophagy exerts its effects on IL-23 (55). In our experiments, in response to LPS/IFN $\gamma, S L A M F 5$-silenced moDCs showed a similar increase of IL- $1 \beta$ and IL-23 production as autophagy-deficient cells, indicating that SLAMF5 may act as a positive regulator of autophagy in moDCs. Our hypothesis that SLAMF5 is a novel cell surface regulator of autophagy was confirmed by two complementary methods; by monitoring conversion of LC3 into its lipid-modified form, and monitoring accumulation of CYTO-ID, an autophagosome-specific dye. In our experiments, however, impaired autophagy was strikingly accompanied by decreased IL-12 secretion, which seems to contradict an earlier study reporting that mice lacking the autophagy protein Atg5 in myeloid cells secrete higher amounts of IL-12 in response to infection with Mtb (56). This discrepancy, however, may be readily resolved by the IRF8 dependence of LPS/IFN $\gamma$-induced IL-12 production in macrophages (14) and, as our results demonstrate, in human moDCs. In addition to IL-12, the transcription of multiple components of the autophagy machinery was recently shown to be under the control of IRF8 in murine DCs. Our work shows that, similarly to murine cells, IRF8 has a pivotal role in the autophagic process of human moDCs. Based on these findings we propose that DC autophagy is controlled by SLAMF5 via an IRF8-dependent mechanism. In support of this, we presented evidence that cross-linking with a SLAMF5-specific agonistic antibody significantly increased the autophagic flux of human moDCs both under steady-state conditions and following activation with LPS/IFN $\gamma$. Importantly, this effect was reversed by silencing IRF8 expression of moDCs. Several studies have demonstrated the importance of posttranslational modifications in the regulation of IRF8 activity $(15,17)$. Pretreatment with the proteasome inhibitor MG132 restored IRF8 protein levels of SLAMF5-silenced cells indicating that in moDCs IRF8 is at least in part controlled by SLAMF5 via modulating its degradation by the proteasome. 
Taken together, data presented in this work strongly suggest that SLAMF5 controls the production of key inflammatory cytokines via regulating autophagy, mediated at least in part by fine-tuning proteasomal degradation of one of the autophagy master regulators, IRF8. Regarding the molecular machinery involved in the described SLAMF5-dependent increase of IRF8 stability, we identified the E3-ubiquitin ligase TRIM21 as a mediator of IRF8 degradation in SLAMF5-silenced moDCs.

However compelling the mechanism of IRF8 control by the SLAMF5 signaling pathway in the regulation of autophagy and inflammatory cytokine production regulation seems, we cannot exclude contribution of other pathways. For example, similar to the mechanism described for SLAMF1 and SLAMF4, SLAMF5 may partake in the formation of the Beclin-1/Vps34 autophagyassociated regulatory complex (22-24). Although much of the molecular details of autophagy regulation are yet to be clarified, SLAMF5 should influence the early events of autophagy, steps independent of autophagosome maturation as well as mTOR, as neither BafA1 nor rapamycin rescued the defect seen in SLAMF5silenced moDCs.

Pharmacological modulation of the autophagy process is of great significance (57). The close to ubiquitous expression of SLAMF5 in hematopoietic cells provides an opportunity to selectively augment autophagic functions in these cells. Immune cell-specific autophagy regulators on one hand could decrease the unwanted pleiotropic effects associated with manipulation of systemic autophagy, and at the same time, increase cell-extrinsic effects of autophagy that operate in the context of host defense.

MoDCs have been reported to be present in the mammalian brain during infections controlling reactive T-lymphocyte influx and contributing to neurodegenerative processes (58). Furthermore, moDCs are also thought to be crucial in brain immunosurveillance, and key players in various neuropathologies associated with Alzheimer's disease (AD) and Parkinson's disease (59). In $\mathrm{AD}$, impaired clearance of amyloid- $\beta$ plaques and tau protein tangles was shown in an SLAMF receptor and Beclin-1 interactome-dependent manner reflecting on the critical involvement of defective autophagy and phagocytosis in $\mathrm{AD}$ pathogenesis (48).

Based on the central role of autophagy in antimicrobial immunity and inflammation, autophagy upregulation in immune cells can be beneficial in infectious diseases (56). In this regard, Garcia et al. have recently reported that autophagy was induced by Mtb-derived antigens in the adherent population of PBMC of Mtb carriers. The level of autophagy was IFN $\gamma$ dependent and showed a positive correlation with the level of IFN $\gamma$ produced by the patient's PBMC (60). Moreover, the same group has shown earlier that $\mathrm{T}$ cells isolated from patients with favorable clinical response to $\mathrm{Mtb}$ (high responders) had increased expression of SLAMF1 compared with those derived from low responders $(61,62)$. Interestingly, they also demonstrated that IL-17 and SLAMF1 have opposing effects on IFN $\gamma$ production in tuberculosis patients (62). These findings are in line with our earlier results in LPS-activated moDCs showing a positive effect of SLAMF1 on the IL-12-IFN $\gamma$ axis in the context of Th1-cell differentiation (63). In this article, we report that SLAMF5 modifies the cytokine profile of LPS/IFN $\gamma$-stimulated moDCs in a manner that would support Th1 cell differentiation in expense to Th17 cell differentiation. However, this interpretation may be overly simplistic. As described by Schmitt et al., IL-12 controls the differentiation of IL-21-producing Tfh-like cells, and induces IL-21 secretion from memory $\mathrm{CD}^{+} \mathrm{T}$ cells (64). These findings, together with the well documented role of SLAMF5 in the differentiation of murine Tfh cells imply that SLAMF5 in human DCs may also promote commitment of naive $\mathrm{CD}^{+} \mathrm{T}$ cells toward the $\mathrm{Tfh}$ lineage. Altogether, our findings suggest that in human DCs, SLAMF5 supports intracellular pathogen-induced immune responses by both increasing the level of autophagy and by modulation of T-cell differentiation. Further research is required to understand the complexity of the underlying mechanisms, and to establish, to what extent, modulation of autophagy using agonistic SLAMF5specific antibodies may be used to improve current therapies for various human diseases.

\section{AUTHOR CONTRIBUTIONS}

$\mathrm{AL}$ and ZA designed the study. ZA, DB, KP, AS, and AL performed experiments. Statistical analysis was done by ZA; confocal microscopy by GV. TB, AB, ER, and PE contributed with essential reagents and made significant conceptual contributions to the project. ZA, PE, and AL wrote the article. All the authors reviewed and approved the final version of the manuscript.

\section{FUNDING}

This work was supported by the National Research, Development and Innovation Office (NKFIH, K-81676 and K-109444, both to $\mathrm{AL})$, the Romanian Ministry of Education, Executive Agency For Higher Education, Research, Development and Innovation Funding, PNCDI II, project no. 119/2014 (to AL, AS, AB, and $\mathrm{ER}$ ), and the GINOP-2.3.2-15-2016-00050 project (to TB, AB, and GV). The project is cofinanced by the European Union and the European Regional Development Fund. This work was also kindly backed by the COST Action BM1404 Mye-EUNITER (www.mye-euniter.eu). COST is supported by the EU Framework Program Horizon 2020 (to ER and AL). KP was supported by the János Bolyai Research Scholarship from the Hungarian Academy of Sciences.

\section{SUPPLEMENTARY MATERIAL}

The Supplementary Material for this article can be found online at http://www.frontiersin.org/articles/10.3389/fimmu.2018.00062/ full\#supplementary-material.

FIGURE S1 | Phenotypic maturation of SLAMF5-silenced moDCs. (A) moDCs were exposed to $100 \mathrm{ng} \mathrm{ml}^{-1} \mathrm{LPS}$ and $10 \mathrm{ng} \mathrm{ml}^{-1} \mathrm{IFN \gamma}$ for $24 \mathrm{~h}$. Cell surface expression of HLA-A, B, C, HLA-DQ, CD40, and CD86 was measured by flow cytometry; values are expressed as reléative fluorescence. Expression of the above cell surface receptors was measured on the $\mathrm{CD1}^{+}$(B) and CD1a- $\mathbf{( C )}$ subsets of control and SLAMF5-silenced moDCs. Bars show the relative fluorescence intensity values (D) Data are presented as means \pm SD of three independent experiments. ( ${ }^{\star} p<0.05$ and ${ }^{\star \star \star} p<0.001$; ns, not significant).

FIGURE S2 | Additional information to SLAMF5-mediated enhancement of dendritic cell autophagy. (A) This panel shows the uncropped version of 
Figure 2A. (B) Analysis of basal level of autophagy in control and SLAMF5silenced cells. Control and SLAMF5-silenced moDCs were left untreated or incubated with $20 \mathrm{nM}$ bafilomycin A1 (BafA1) for $2 \mathrm{~h}$. LC3 and $\beta$-actin levels were analyzed by western blotting. In this panel, samples of BafA1-treated and untreated cells are run on the same gel. Please note the robust accumulation of LC3-II in the BafA1-treated samples! BafA1-treated samples are badly overexposed precluding quantitation of LC3-II readouts of BafA1-treated and untreated samples from the same gel. Thus, samples derived from BafA1-treated and untreated samples were run and analyzed separately. Of note, at shorter exposure times, LC3-I band is not visible on blots of the BafA1-treated samples. (C) Autophagosome formation was visualized by CYTO-ID staining followed by confocal microscopy in control and SLAMF5-silenced moDCs in the presence or absence of $50 \mu \mathrm{M}$ Chloroquine, an inhibitor of lysosomal acidification. Representative images show appearance of fluorescent puncta due to vacuolar localization of CYTO-ID in autophagosomes. Nuclei are highlighted by Hoechst 33342 staining. FOV: $57.4 \mu \mathrm{m} \times 44.9 \mu \mathrm{m}$.

FIGURE S3 | SLAMF5 expression in IRF8 knockdown moDCs. On day 5 of moDC differentiation, cell surface expression of SLAMF5 in IRF8-silenced or control moDCs was measured by flow cytometry. Values are expressed as

\section{REFERENCES}

1. Kuma A, Mizushima N. Physiological role of autophagy as an intracellular recycling system: with an emphasis on nutrient metabolism. Semin Cell Dev Biol (2010) 21(7):683-90. doi:10.1016/j.semcdb.2010.03.002

2. Guo JY, Teng X, Laddha SV, Ma S, Van Nostrand SC, Yang Y, et al. Autophagy provides metabolic substrates to maintain energy charge and nucleotide pools in Ras-driven lung cancer cells. Genes Dev (2016) 30(15):1704-17. doi:10.1101/gad.283416.116

3. Green DR, Galluzzi L, Kroemer G. Mitochondria and the autophagyinflammation-cell death axis in organismal aging. Science (2011) 333(6046): 1109-12. doi:10.1126/science. 1201940

4. Munz C. Enhancing immunity through autophagy. Annu Rev Immunol (2009) 27:423-49. doi:10.1146/annurev.immunol.021908.132537

5. Deretic V, Kimura T, Timmins G, Moseley P, Chauhan S, Mandell M. Immunologic manifestations of autophagy. J Clin Invest (2015) 125(1):75-84. doi:10.1172/JCI73945

6. Cadwell K. Crosstalk between autophagy and inflammatory signalling pathways: balancing defence and homeostasis. Nat Rev Immunol (2016) 16(11):661-75. doi:10.1038/nri.2016.100

7. Varol C, Landsman L, Fogg DK, Greenshtein L, Gildor B, Margalit R, et al. Monocytes give rise to mucosal, but not splenic, conventional dendritic cells. J Exp Med (2007) 204(1):171-80. doi:10.1084/jem.20061011

8. Greter M, Helft J, Chow A, Hashimoto D, Mortha A, Agudo-Cantero J, et al. GM-CSF controls nonlymphoid tissue dendritic cell homeostasis but is dispensable for the differentiation of inflammatory dendritic cells. Immunity (2012) 36(6):1031-46. doi:10.1016/j.immuni.2012.03.027

9. Zhang Y, Morgan MJ, Chen K, Choksi S, Liu ZG. Induction of autophagy is essential for monocyte-macrophage differentiation. Blood (2012) 119(12):2895-905. doi:10.1182/blood-2011-08-372383

10. Schmid D, Pypaert M, Munz C. Antigen-loading compartments for major histocompatibility complex class II molecules continuously receive input from autophagosomes. Immunity (2007) 26(1):79-92. doi:10.1016/j.immuni. 2006.10.018

11. Duraes FV, Niven J, Dubrot J, Hugues S, Gannage M. Macroautophagy in endogenous processing of self- and pathogen-derived antigens for MHC class II presentation. Front Immunol (2015) 6:459. doi:10.3389/fimmu.2015.00459

12. Terawaki S, Camosseto V, Prete F, Wenger T, Papadopoulos A, Rondeau C, et al. RUN and FYVE domain-containing protein 4 enhances autophagy and lysosome tethering in response to interleukin-4. JCell Biol (2015) 210(7):1133-52. doi:10.1083/jcb.201501059

13. Gupta M, Shin DM, Ramakrishna L, Goussetis DJ, Platanias LC, Xiong H, et al. IRF8 directs stress-induced autophagy in macrophages and promotes clearance of Listeria monocytogenes. Nat Commun (2015) 6:6379. doi:10.1038/ ncomms 7379 relative fluorescence and are the mean $+\mathrm{SD}$ of five independent experiments $\left({ }^{*} p<0.05\right)$.

FIGURE S4 | Accumulation of ubiquitinated proteins in the presence of the proteasome inhibitor MG132. This panel shows a representative blot of control experiments to confirm the activity of MG132 in our experimental system. MoDCs were treated as described in the legend for Figure 5B. Western blot was developed by a ubiquitin-specific antibody as described in Section "Materials and Methods."

FIGURE S5 | TRIM21 expression is enhanced in response to treatment with LPS/IFN $\gamma$. SLAMF5 knockdown or control moDCs were exposed to $100 \mathrm{ng} \mathrm{ml}^{-1}$ LPS and $10 \mathrm{ng} \mathrm{ml}^{-1}$ IFN $\gamma$ for $8 \mathrm{~h}$. TRIM21 protein levels were determined by western blot analysis. A representative of three independent experiments is shown. Bar chart on the right displays TRIM21 protein levels of SLAMF5-silenced or control moDCs relative to $\beta$-actin ( ${ }^{*} p<0.05$ ).

FIGURE S6 | EAT-2 expression levels in human monocytes and moDCs. Western blot analysis of EAT-2 expression in monocytes and moDCs on day 5 of in vitro differentiation treated or not with LPS/IFN $\gamma$. A representative blot of three independent experiments is shown,

with $\beta$-actin as loading control.

14. Wang IM, Contursi C, Masumi A, Ma X, Trinchieri G, Ozato K. An IFNgamma-inducible transcription factor, IFN consensus sequence binding protein (ICSBP), stimulates IL-12 p40 expression in macrophages. J Immunol (2000) 165(1):271-9. doi:10.4049/jimmunol.165.1.271

15. Kong HJ, Anderson DE, Lee CH, Jang MK, Tamura T, Tailor P, et al. Cutting edge: autoantigen Ro52 is an interferon inducible E3 ligase that ubiquitinates IRF-8 and enhances cytokine expression in macrophages. J Immunol (2007) 179(1):26-30. doi:10.4049/jimmunol.179.1.26

16. Kim JY, Ozato K. The sequestosome 1/p62 attenuates cytokine gene expression in activated macrophages by inhibiting IFN regulatory factor 8 and TNF receptor-associated factor 6/NF-kappaB activity. JImmunol (2009) 182(4):2131-40. doi:10.4049/jimmunol.0802755

17. Xiong H, Li H, Kong HJ, Chen Y, Zhao J, Xiong S, et al. Ubiquitin-dependent degradation of interferon regulatory factor- 8 mediated by $\mathrm{Cbl}$ down-regulates interleukin-12 expression. J Biol Chem (2005) 280(25):23531-9. doi:10.1074/ jbc.M414296200

18. Lin R, Nie J, Ren J, Liang R, Li D, Wang P, et al. USP4 interacts and positively regulates IRF8 function via K48-linked deubiquitination in regulatory $\mathrm{T}$ cells. FEBS Lett (2017) 591(12):1677-86. doi:10.1002/1873-3468.12668

19. Cannons JL, Tangye SG, Schwartzberg PL. SLAM family receptors and SAP adaptors in immunity. Annu Rev Immunol (2011) 29:665-705. doi:10.1146/ annurev-immunol-030409-101302

20. Ma CS, Nichols KE, Tangye SG. Regulation of cellular and humoral immune responses by the SLAM and SAP families of molecules. Annu Rev Immunol (2007) 25:337-79. doi:10.1146/annurev.immunol.25.022106.141651

21. van Driel BJ, Liao G, Engel P, Terhorst C. Responses to microbial challenges by SLAMF receptors. Front Immunol (2016) 7:4. doi:10.3389/fimmu. 2016.00004

22. Berger SB, Romero X, Ma C, Wang G, Faubion WA, Liao G, et al. SLAM is a microbial sensor that regulates bacterial phagosome functions in macrophages. Nat Immunol (2010) 11(10):920-7. doi:10.1038/ni.1931

23. Chaudhary A, Leite M, Kulasekara BR, Altura MA, Ogahara C, Weiss E, et al. Human diversity in a cell surface receptor that inhibits autophagy. Curr Biol (2016) 26(14):1791-801. doi:10.1016/j.cub.2016.05.003

24. Ma C, Wang N, Detre C, Wang G, O'Keeffe M, Terhorst C. Receptor signaling lymphocyte-activation molecule family 1 (Slamf1) regulates membrane fusion and NADPH oxidase 2 (NOX2) activity by recruiting a Beclin-1/Vps34/ultraviolet radiation resistance-associated gene (UVRAG) complex. J Biol Chem (2012) 287(22):18359-65. doi:10.1074/jbc.M112. 367060

25. Bologna C, Buonincontri R, Serra S, Vaisitti T, Audrito V, Brusa D, et al. SLAMF1 regulation of chemotaxis and autophagy determines CLL patient response. J Clin Invest (2016) 126(1):181-94. doi:10.1172/JCI83013

26. Tangye SG, van de Weerdt BC, Avery DT, Hodgkin PD. CD84 is up-regulated on a major population of human memory $\mathrm{B}$ cells and recruits the $\mathrm{SH} 2$ domain 
containing proteins SAP and EAT-2. Eur J Immunol (2002) 32(6):1640-9. doi:10.1002/1521-4141(200206)32:6<1640:AID-IMMU 1640>3.0. CO;2-S

27. Martin M, Romero X, de la Fuente MA, Tovar V, Zapater N, Esplugues E, et al. CD84 functions as a homophilic adhesion molecule and enhances IFN-gamma secretion: adhesion is mediated by Ig-like domain 1. J Immunol (2001) 167(7):3668-76. doi:10.4049/jimmunol.167.7.3668

28. Cannons JL, Qi H, Lu KT, Dutta M, Gomez-Rodriguez J, Cheng J, et al. Optimal germinal center responses require a multistage $\mathrm{T}$ cell: $\mathrm{B}$ cell adhesion process involving integrins, SLAM-associated protein, and CD84. Immunity (2010) 32(2):253-65. doi:10.1016/j.immuni.2010.01.010

29. Romero X, Benitez D, March S, Vilella R, Miralpeix M, Engel P. Differential expression of SAP and EAT-2-binding leukocyte cell-surface molecules CD84, CD150 (SLAM), CD229 (Ly9) and CD244 (2B4). Tissue Antigens (2004) 64(2):132-44. doi:10.1111/j.1399-0039.2004.00247.x

30. Mathan TS, Figdor CG, Buschow SI. Human plasmacytoid dendritic cells: from molecules to intercellular communication network. Front Immunol (2013) 4:372. doi:10.3389/fimmu.2013.00372

31. Binsky-Ehrenreich I, Marom A, Sobotta MC, Shvidel L, Berrebi A, HazanHalevy I, et al. CD84 is a survival receptor for CLL cells. Oncogene (2014) 33(8):1006-16. doi:10.1038/onc.2013.31

32. Kabeya Y, Mizushima N, Ueno T, Yamamoto A, Kirisako T, Noda T, et al. LC3, a mammalian homologue of yeast Apg8p, is localized in autophagosome membranes after processing. EMBO J (2000) 19(21):5720-8. doi:10.1093/ emboj/19.21.5720

33. Mizushima N, Yoshimori T. How to interpret LC3 immunoblotting. Autophagy (2007) 3(6):542-5. doi:10.4161/auto.4600

34. Mauvezin C, Neufeld TP. Bafilomycin A1 disrupts autophagic flux by inhibiting both V-ATPase-dependent acidification and Ca-P60A/SERCAdependent autophagosome-lysosome fusion. Autophagy (2015) 11(8):1437-8. doi:10.1080/15548627.2015.1066957

35. Chan LL, Shen D, Wilkinson AR, Patton W, Lai N, Chan E, et al. A novel image-based cytometry method for autophagy detection in living cells. Autophagy (2012) 8(9):1371-82. doi:10.4161/auto.21028

36. van Loosdregt J, Rossetti M, Spreafico R, Moshref M, Olmer M, Williams GW, et al. Increased autophagy in CD4+ T cells of rheumatoid arthritis patients results in T-cell hyperactivation and apoptosis resistance. Eur J Immunol (2016) 46(12):2862-70. doi:10.1002/eji.201646375

37. Oeste CL, Seco E, Patton WF, Boya P, Perez-Sala D. Interactions between autophagic and endo-lysosomal markers in endothelial cells. Histochem Cell Biol (2013) 139(5):659-70. doi:10.1007/s00418-012-1057-6

38. Tangye SG, Nichols KE, Hare NJ, van de Weerdt BC. Functional requirements for interactions between CD84 and Src homology 2 domain-containing proteins and their contribution to human T cell activation. J Immunol (2003) 171(5):2485-95. doi:10.4049/jimmunol.171.5.2485

39. Jung $\mathrm{CH}$, Ro $\mathrm{SH}$, Cao J, Otto NM, Kim DH. mTOR regulation of autophagy. FEBS Lett (2010) 584(7):1287-95. doi:10.1016/j.febslet.2010.01.017

40. Granato M, Lacconi V, Peddis M, Di Renzo L, Valia S, Rivanera D, et al. Hepatitis $C$ virus present in the sera of infected patients interferes with the autophagic process of monocytes impairing their in-vitro differentiation into dendritic cells. Biochim Biophys Acta (2014) 1843(7):1348-55. doi:10.1016/j. bbamcr.2014.04.003

41. Calpe S, Wang N, Romero X, Berger SB, Lanyi A, Engel P, et al. The SLAM and SAP gene families control innate and adaptive immune responses. Adv Immunol (2008) 97:177-250. doi:10.1016/S0065-2776(08) 00004-7

42. Morra M, Lu J, Poy F, Martin M, Sayos J, Calpe S, et al. Structural basis for the interaction of the free SH2 domain EAT-2 with SLAM receptors in hematopoietic cells. EMBO J (2001) 20(21):5840-52. doi:10.1093/emboj/ 20.21.5840

43. Roncagalli R, Taylor JE, Zhang S, Shi X, Chen R, Cruz-Munoz ME, et al. Negative regulation of natural killer cell function by EAT-2, a SAP-related adaptor. Nat Immunol (2005) 6(10):1002-10. doi:10.1038/ni1242

44. Wang A, Batteux F, Wakeland EK. The role of SLAM/CD2 polymorphisms in systemic autoimmunity. Curr Opin Immunol (2010) 22(6):706-14. doi:10.1016/j. coi.2010.10.014
45. van Driel B, Liao G, Romero X, O'Keeffe MS, Wang G, Faubion WA, et al. Signaling lymphocyte activation molecule regulates development of colitis in mice. Gastroenterology (2012) 143(6):1544-54.e7. doi:10.1053/j.gastro. 2012.08.042

46. $\mathrm{Wu} \mathrm{N}$, Veillette A. SLAM family receptors in normal immunity and immune pathologies. Curr Opin Immunol (2016) 38:45-51. doi:10.1016/j. coi.2015.11.003

47. Yang C, Hu G, Li Z, Wang Q, Wang X, Yuan C, et al. Differential gene expression in patients with subsyndromal symptomatic depression and major depressive disorder. PLoS One (2017) 12(3):e0172692. doi:10.1371/journal. pone. 0172692

48. Salminen A, Kaarniranta K, Kauppinen A, Ojala J, Haapasalo A, Soininen H, et al. Impaired autophagy and APP processing in Alzheimer's disease: the potential role of Beclin 1 interactome. Prog Neurobiol (2013) 10(6-107):33-54. doi:10.1016/j.pneurobio.2013.06.002

49. Alvarez-Errico D, Oliver-Vila I, Ainsua-Enrich E, Gilfillan AM, Picado C, Sayos J, et al. CD84 negatively regulates IgE high-affinity receptor signaling in human mast cells. J Immunol (2011) 187(11):5577-86. doi:10.4049/jimmunol. 1101626

50. Sintes J, Romero X, de Salort J, Terhorst C, Engel P. Mouse CD84 is a pan-leukocyte cell-surface molecule that modulates LPS-induced cytokine secretion by macrophages. J Leukoc Biol (2010) 88(4):687-97. doi:10.1189/ jlb.1109756

51. Napolitani G, Bortoletto N, Racioppi L, Lanzavecchia A, D’Oro U. Activation of src-family tyrosine kinases by LPS regulates cytokine production in dendritic cells by controlling AP-1 formation. Eur J Immunol (2003) 33(10): 2832-41. doi:10.1002/eji.200324073

52. Kuka M, Baronio R, Valentini S, Monaci E, Muzzi A, Aprea S, et al. Src kinases are required for a balanced production of IL-12/IL-23 in human dendritic cells activated by toll-like receptor agonists. PLoS One (2010) 5(7):e11491. doi:10.1371/journal.pone.0011491

53. Crisan TO, Plantinga TS, van de Veerdonk FL, Farcas MF, Stoffels M, Kullberg BJ, et al. Inflammasome-independent modulation of cytokine response by autophagy in human cells. PLoS One (2011) 6(4):e18666. doi:10.1371/journal. pone.0018666

54. Kleinnijenhuis J, Oosting M, Plantinga TS, van der Meer JW, Joosten LA, Crevel RV, et al. Autophagy modulates the Mycobacterium tuberculosisinduced cytokine response. Immunology (2011) 134(3):341-8. doi:10.1111/j. 1365-2567.2011.03494.x

55. Peral de Castro C, Jones SA, Ni Cheallaigh C, Hearnden CA, Williams L, Winter J, et al. Autophagy regulates IL-23 secretion and innate $\mathrm{T}$ cell responses through effects on IL-1 secretion. JImmunol (2012) 189(8):4144-53. doi:10.4049/jimmunol.1201946

56. Castillo EF, Dekonenko A, Arko-Mensah J, Mandell MA, Dupont N, Jiang S, et al. Autophagy protects against active tuberculosis by suppressing bacterial burden and inflammation. Proc Natl Acad Sci U S A (2012) 109(46): E3168-76. doi:10.1073/pnas.1210500109

57. Galluzzi L, Bravo-San Pedro JM, Levine B, Green DR, Kroemer G. Pharmacological modulation of autophagy: therapeutic potential and persisting obstacles. Nat Rev Drug Discov (2017) 16(7):487-511. doi:10.1038/nrd. 2017.22

58. Hirako IC, Ataide MA, Faustino L, Assis PA, Sorensen EW, Ueta H, et al. Splenic differentiation and emergence of CCR5+CXCL9+CXCL10+ monocyte-derived dendritic cells in the brain during cerebral malaria. Nat Commun (2016) 7:13277. doi:10.1038/ncomms13277

59. Bossu P, Spalletta G, Caltagirone C, Ciaramella A. Myeloid dendritic cells are potential players in human neurodegenerative diseases. Front Immunol (2015) 6:632. doi:10.3389/fimmu.2015.00632

60. Rovetta AI, Pena D, Hernandez Del Pino RE, Recalde GM, Pellegrini J, Bigi F, et al. IFNG-mediated immune responses enhance autophagy against Mycobacterium tuberculosis antigens in patients with active tuberculosis. Autophagy (2014) 10(12):2109-21. doi:10.4161/15548627.2014.981791

61. Quiroga MF, Martinez GJ, Pasquinelli V, Costas MA, Bracco MM, Malbran A, et al. Activation of signaling lymphocytic activation molecule triggers a signaling cascade that enhances Th1 responses in human intracellular infection. J Immunol (2004) 173(6):4120-9. doi:10.4049/jimmunol.173.6.4120 
62. Pasquinelli V, Townsend JC, Jurado JO, Alvarez IB, Quiroga MF, Barnes PF, et al. IFN-gamma production during active tuberculosis is regulated by mechanisms that involve IL-17, SLAM, and CREB. J Infect Dis (2009) 199(5):661-5. doi:10.1086/596742

63. Rethi B, Gogolak P, Szatmari I, Veres A, Erdos E, Nagy L, et al. SLAM/SLAM interactions inhibit CD40-induced production of inflammatory cytokines in monocyte-derived dendritic cells. Blood (2006) 107(7):2821-9. doi:10.1182/ blood-2005-06-2265

64. Schmitt N, Morita R, Bourdery L, Bentebibel SE, Zurawski SM, Banchereau J, et al. Human dendritic cells induce the differentiation of interleukin-21producing $\mathrm{T}$ follicular helper-like cells through interleukin-12. Immunity (2009) 31(1):158-69. doi:10.1016/j.immuni.2009.04.016
Conflict of Interest Statement: The authors declare that the research was conducted in the absence of any commercial or financial relationships that could be construed as a potential conflict of interest.

The reviewer MG and handling editor declared their shared affiliation.

Copyright (c) 2018 Agod, Pazmandi, Bencze, Vereb, Biro, Szabo, Rajnavolgyi, Bacsi, Engel and Lanyi. This is an open-access article distributed under the terms of the Creative Commons Attribution License (CC BY). The use, distribution or reproduction in other forums is permitted, provided the original author(s) and the copyright owner are credited and that the original publication in this journal is cited, in accordance with accepted academic practice. No use, distribution or reproduction is permitted which does not comply with these terms. 